\title{
Modeling and Control of 2D Grasping under Rolling Contact Constraints between Arbitrary Shapes: A Riemannian-Geometry Approach
}

\author{
Suguru Arimoto ${ }^{1,2}$ and Morio Yoshida ${ }^{2}$ \\ ${ }^{1}$ Research Organization of Science and Engineering, Ritsumeikan University, Kusatsu, Shiga 525-8577, Japan \\ ${ }^{2}$ RIKEN-TRI Collaboration, Center for Human-Interactive Robot Research, Nagoya, Aichi 463-0003, Japan \\ Correspondence should be addressed to Suguru Arimoto, arimoto@fc.ritsumei.ac.jp
}

Received 16 July 2009; Revised 7 December 2009; Accepted 19 January 2010

Academic Editor: Warren Dixon

Copyright (C) 2010 S. Arimoto and M. Yoshida. This is an open access article distributed under the Creative Commons Attribution License, which permits unrestricted use, distribution, and reproduction in any medium, provided the original work is properly cited.

\begin{abstract}
Modeling, control, and stabilization of dynamics of two-dimensional object grasping by using a pair of multijoint robot fingers are investigated under rolling contact constraints and arbitrariness of the geometry of the object and fingertips. First, modeling of rolling motion between 2D rigid bodies with arbitrary shape is treated under the assumption that the two contour curves coincide at the contact point and share the same tangent. The rolling constraints induce the Euler equation of motion that is parameterized by a pair of arclength parameters and constrained onto the kernel space as an orthogonal complement to the image space spanned from all the constraint gradients. Furthermore, it is shown that all the Pfaffian forms of the rolling constraints are integrable in the sense of Frobenius and therefore the rolling contacts are regarded as a holonomic constraint. The Euler-Lagrange equation of motion of the overall fingers/object system is rederived together with a couple of first-order differential equations that express evolution of contact points in terms of quantities of the second fundamental form. A control signal called "blind grasping" is defined and shown to be effective in maintenance or stabilization of grasping without using the details of object shape and parameters or external sensing. An extension of the Dirichlet-Lagrange stability theorem to a system of DOF-redundancy under constraints is discussed by introducing a Morse-Bott function and deriving its Hessian, in a special case that the object to be grasped is a parallelepiped.
\end{abstract}

\section{Introduction}

This paper aims at tackling the control problem for dextrous multifingered hands from computational perspectives (see Figure 1), based on the assumption that a complete model of grasping must be developed even under the existence of rolling contacts and the arbitrariness of geometry of objects. So far the kinematics and geometry of contact between rigid bodies were solved by Montanna [1], and a set of velocity relations is given in detail [1]. However, there is a dearth of papers except the papers mentioned in [2-4] that attempt to model dynamics of physical interactions between the fingerends and an object under the existence of rollings. However, the papers [2-4] did not yet gain physical insights into the constraint forces arising from rolling contacts and show any explicit forms of them in the object wrench space.
In the series of our papers $[5,6]$, a set of Lagrange equations of motion of the overall fingers/object system under rolling constraints is derived under the assumption that rolling is interpreted as a constraint of the equal velocity of the contact point running on the fingerend sphere relative to running on the object surface, as originally formulated in the text book [7]. However, all discussions in $[5,6]$ have been restricted to the case of ball-plate rollings. Very recently, a complete model of 2-dimensional grasping of a rigid object with arbitrary shape is given as a set of Lagrange's equations of motion of the overall fingers/object system together with a pair of firstorder differential equations that update arclength parameters [8]. It should be noted that modeling of the system assumes the full knowledge of geometry of a given object but design of control signals neither needs the information of object geometry nor uses any external sensing of contact points. 


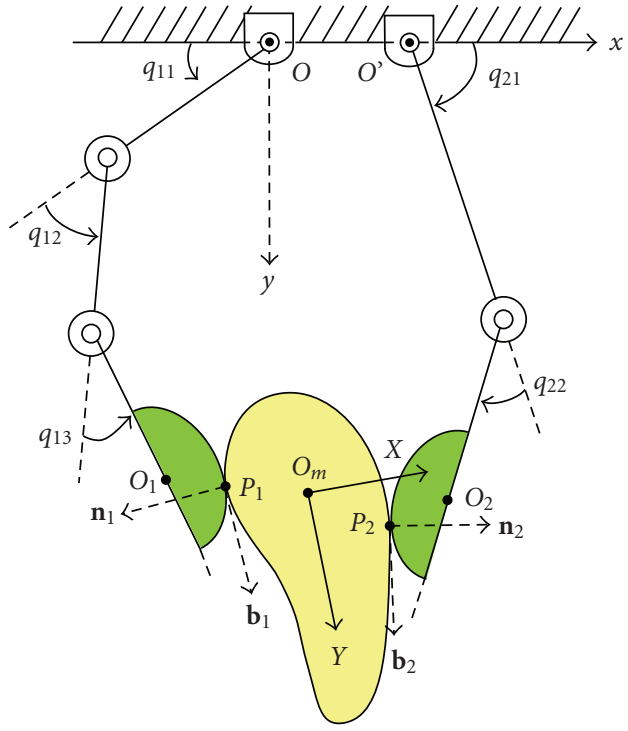

FIgURE 1: A pair of two-dimensional robot fingers with a curved fingertip makes rolling contact with a rigid object with a curved contour.

This paper further presents a complete model of grasping in the case that the geometry of fingerends is arbitrary and discusses a control scheme called "blind control" that need neither the use the geometric information of the fingerends and object nor the sense locations of the contact points and object mass center. In this paper, rolling contact is interpreted as the following conditions.

(1) Two contact points on the contour curves must coincide at a single point.

(2) The two contours must have the same tangent at the common contact point.

These two conditions as a whole are equivalent to Nomizu's relation [9] concerning tangent vectors at the contact point and normals to the common tangent. The argument presented in [9] can be interpreted as a kinematic extension of the well-known theorem on curves that if two smooth curves have the same curvatures along their arclengths then the two curves can be exactly superposed by using a homogeneous transformation. By virtue of this mathematical standpoint of rolling contact, we can show immediately that the Euler-Lagrange equation of motion of the overall fingers/object system is characterized by a pair of arclength parameters and quantities of only the first fundamental form of contour curves. It is further shown that the Euler-Lagrange equation should be accompanied with a pair of first-order differential equations that govern evolutions of arclength parameters, where quantities of the second fundamental form are involved. Furthermore, we show that rolling contact constraints expressed in terms of velocity relations are integrable in the sense of Frobenius. In other words, the rolling contact constraints can be regarded as a holonomic constraint. Based upon these holonomic constraint forms, we reformulate the Euler-Lagrange equation of motion of the system.
In the final two sections, we will discuss an extension of Dirichlet-Lagrange stability theorem from the standpoint of Riemannian Geometry by introducing a control signal called "blind grasping." The signal can be constructed realtime based upon the data of fingers' kinematics without referring to object kinematics, regarding the pair of robot fingers as the intrinsic world but objects to be grasped existing in the external world. We show that the blind control signal designed on the basis of the fingers-thumb opposability can be interpreted as a Morse-Bott function in the case that the object is a parallelepiped, since its Hessina in this case becomes positive semidefinite on the base manifold. Notwithstanding the redundancy in system's DOF, we prove the asymptotic convergence of a solution trajectory to the closed-loop dynamics to an equilibrium manifold that attains minimization of the Morse-Bott function. We finally point out a few of important and interesting problems that remain unsolved or not yet tackled.

\section{Definitions of Tangent Vectors in Terms of Arclength Parameters}

First, denote the contour curve of the left hand fingertip by $\gamma_{1}\left(s_{1}\right)$ in terms of arclength parameter $s_{1}$ as shown in Figure 2 and the object contour by $\gamma_{01}\left(s_{0}\right)$ in terms of another length parameter $s_{0}$. Suppose that the fingertip contour contacts with the object at $\gamma_{1}\left(s_{1}\right)=P_{1 A}$ and $\gamma_{01}\left(s_{0}\right)=P_{1 B}$ so that $P_{1 A}=P_{1 B}=P_{1}$ in the frame coordinate space. In general, we assume implicitly that by rolling motion the contact point $P_{1}$ moves on each contour with the same run-length without splipping. Therefore, we characterize the contour curves $\gamma_{1}(s)$ and $\gamma_{01}(s)$ by using the same length parameter $s$, at which $P_{1 A}(s)$ and $P_{1 B}(s)$ expressed in the frame space $O-$ $x y$ coincide and share the same tangent vector, as shown in Figures 2 and 3.

In order to gain a physical insight into this rolling constraint, we need to denote firstly the fingertip contour curve $\gamma_{1}(s)$ in the local coordinates $O_{1}-X_{1} Y_{1}$ as shown in Figure 3 and the object contour by $\gamma_{01}(s)$ in the object coordinates $O_{m}-X Y$ fixed at the object. If we denote $\gamma_{1}(s)$ and $\gamma_{01}(s)$ by

$$
\gamma_{1}(s)=\left(\begin{array}{c}
X_{1}(s) \\
Y_{1}(s)
\end{array}\right), \quad \gamma_{01}(s)=\left(\begin{array}{c}
X_{01}(s) \\
Y_{01}(s)
\end{array}\right)
$$

and define

$$
\begin{gathered}
\mathbf{b}_{1}(s)=\gamma_{1}^{\prime}(s)=\frac{\mathrm{d}}{\mathrm{d} s}\left(\begin{array}{c}
X_{1}(s) \\
Y_{1}(s)
\end{array}\right), \\
\mathbf{b}_{01}(s)=\gamma_{01}^{\prime}(s)=\frac{\mathrm{d}}{\mathrm{d} s}\left(\begin{array}{c}
X_{01}(s) \\
Y_{01}(s)
\end{array}\right),
\end{gathered}
$$

then $\mathbf{b}_{1}(s)$ must be the unit tangent vector at the point $P_{1 A}(s)$ and $\mathbf{b}_{01}(s)$ must be also the unit tangent vector at $P_{1 B}(s)$ (see Figure 3 ). It is well known in the mathematical theory of curves and surfaces that the further derivatives of $\mathbf{b}_{1}$ and $\mathbf{b}_{01}$ in $s$ can be expressed as

$$
\mathbf{b}_{1}^{\prime}(s)=\kappa_{1}(s) \mathbf{n}_{1}(s), \quad \mathbf{b}_{01}^{\prime}(s)=\kappa_{01}(s) \mathbf{n}_{01}(s),
$$




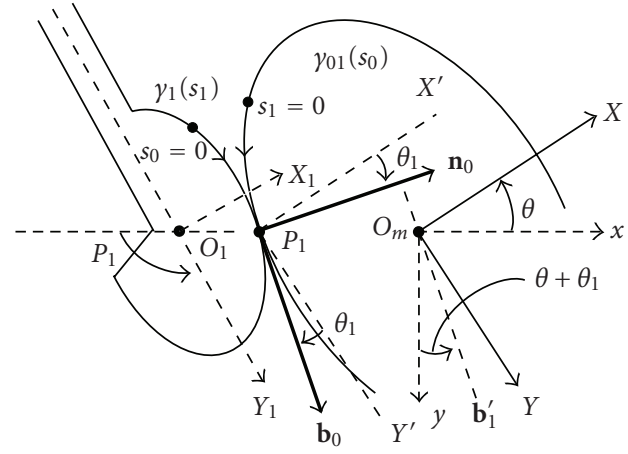

Figure 2: Definitions of local coordinates $O_{m}-X Y$ for the object and $O_{1}-X_{1} Y_{1}$ for the fingertip.

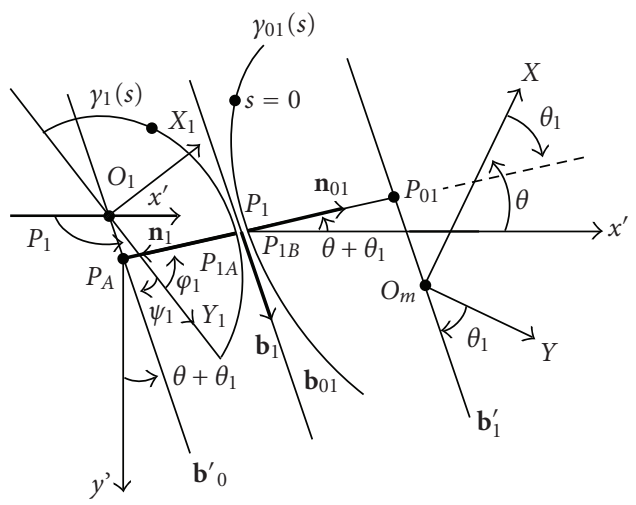

FIGURE 3: Rolling contact constraint is expressed by equalizing the velocity of contact point $P_{1 A}\left(=P_{1}\right)$ in the direction of the unit tangent vector $\mathbf{b}_{0}$ with that of $P_{1 B}\left(=P_{1}\right)$ in the same direction of $\mathbf{b}_{1}$. The velocity of $P_{1 A}$ in the direction of the normal $\mathbf{n}_{1}$ should be equal to that of $P_{1 B}$ in the same direction. It is therefore assumed that $\mathbf{b}_{0}=\mathbf{b}_{1}$.

where $\mathbf{n}_{1}(s)$ and $\mathbf{n}_{01}(s)$ are orthogonal to $\mathbf{b}_{1}(s)$ and $\mathbf{b}_{01}(s)$, respectively, and $\kappa_{1}(s)$ is the curvature of the fingertip contour curve and $\kappa_{01}(s)$ is that of the object contour in its left-hand side. It is also well known that

$$
\mathbf{n}_{1}^{\prime}(s)=-\kappa_{1}(s) \mathbf{b}_{1}(s), \quad \mathbf{n}_{01}^{\prime}(s)=-\kappa_{01}(s) \mathbf{b}_{01}(s),
$$

and this equation together with (4) constitutes the FrenetSerret formula (see [10]).

Next consider the rotation matrix of the object around its mass center $O_{m}$ expressed in the frame coordinates as follows:

$$
\Pi_{0}=\left(\mathbf{r}_{X}, \mathbf{r}_{Y}\right),
$$

where $\mathbf{r}_{X}$ denotes the unit vector of $X$-axis and $\mathbf{r}_{Y}$ that of $Y$-axis expressed in the inertial frame coordinates $O-x y$ as shown in Figure 2. If the object rotates by angle $\theta$, that is, $O_{m}-X Y$ rotates by $\theta$ from the frame coordinates $O-x y$, then

$$
\Pi_{0}=\left(\mathbf{r}_{X}, \mathbf{r}_{Y}\right)=\left(\begin{array}{cc}
\cos \theta & \sin \theta \\
-\sin \theta & \cos \theta
\end{array}\right)
$$

Similarly, define

$$
\Pi_{1}=\left(\mathbf{r}_{X 1}, \mathbf{r}_{Y 1}\right)
$$

where $\mathbf{r}_{X 1}$ denotes the unit vector of $X_{1}$-axis of the lefthand fingertip expressed in the frame coordinates $O-x y$ and $\mathbf{r}_{Y 1}$ that of $Y_{1}$-axis. Both the matrices $\Pi_{0}$ and $\Pi_{1}$ are an orthogonal matrix regarded as an element of $\mathrm{SO}(2)$. The derivatives of $\Pi_{0}$ and $\Pi_{1}$ in $t$ denoted by $\dot{\Pi}_{0}=\left(\mathrm{d} \Pi_{0} / \mathrm{d} t\right)$ and $\dot{\Pi}_{1}$ are obtained as follows:

$$
\begin{aligned}
& \dot{\Pi}_{0}=\Pi_{0} \Omega_{0}, \quad \Omega_{0}=\dot{\theta}\left(\begin{array}{cc}
0 & 1 \\
-1 & 0
\end{array}\right), \\
& \dot{\Pi}_{1}=\Pi_{1} \Omega_{1}, \quad \Omega_{1}=\dot{p}_{1}\left(\begin{array}{cc}
0 & 1 \\
-1 & 0
\end{array}\right),
\end{aligned}
$$

where $p_{1}=q_{11}+q_{12}+q_{13}=q_{1}^{\mathrm{T}} \mathbf{e}_{1}, q_{1}=\left(q_{11}, q_{12}, q_{13}\right)^{\mathrm{T}}$, and $\mathbf{e}=(1,1,1)^{\mathrm{T}}$ in the case of the finger mechanism (a planar robot with three joints) depicted in Figure 1. Throughout the paper, we take the positive sign of angles $\theta, \theta_{i}, p_{i}$, and $q_{i j}$ in the counterclockwise direction. Hence, $\Omega_{0}$ in (9) expresses the rotation of positive angle $\pi / 2$ with associating the angular velocity $\dot{\theta}$. In other words, $\Omega_{0} \mathbf{b}_{01}=\dot{\theta} \mathbf{n}_{01}$ or $\mathbf{b}_{01}^{\mathrm{T}} \Omega_{0}=-\dot{\theta} \mathbf{n}_{01}^{\mathrm{T}}$ and $\Omega_{1} \mathbf{b}_{1}=-\dot{p}_{1} \mathbf{n}_{1}$ or $\mathbf{b}_{1}^{\mathrm{T}} \Omega_{1}=\dot{p}_{1} \mathbf{n}_{1}^{\mathrm{T}}$.

Next, denote the position vector of point $O_{1}$ as a center of the left-hand fingertip by $\mathbf{r}_{1}\left(=\overrightarrow{O_{1}}\right)$ and that of the object mass center by $\mathbf{r}_{m}\left(=\overrightarrow{O O_{m}}\right)$. Then, the first assumption that the two contact points $P_{1 A}$ and $P_{1 B}$ coincide at the common contact point $P_{1}$ implies the equality

$$
\left(\mathbf{r}_{1 A}=\right) \mathbf{r}_{1}+\Pi_{1} \gamma_{1}=\mathbf{r}_{m}+\Pi_{0} \gamma_{01} \quad\left(=\mathbf{r}_{1 B}\right)
$$

For the sake of convenience, we denote the unit tangent vector at $P_{1}$ expressed in the frame space $O-x y$ by

$$
\overline{\mathbf{b}}_{1}=\Pi_{1} \mathbf{b}_{1}=\Pi_{1} \frac{\partial \gamma_{1}}{\partial s}, \quad \overline{\mathbf{b}}_{01}=\Pi_{0} \mathbf{b}_{01}=\Pi_{0} \frac{\partial \gamma_{01}}{\partial s} .
$$

Then, the second assumption that at the contact point the two curves share the same tangent implies

$$
\overline{\mathbf{b}}_{1}=\Pi_{1} \mathbf{b}_{1}=\Pi_{0} \mathbf{b}_{01}=\overline{\mathbf{b}}_{01} \text {. }
$$

Throughout the paper we use symbol ( $\left.{ }^{*}\right)$ for expressing the derivative in $t$ in such a way that $\dot{r}_{1}=\mathrm{d} \mathbf{r}_{1} / \mathrm{d} t$ and $\dot{\Pi}_{1}=$ $\mathrm{d} \Pi_{1} / \mathrm{d} t$. Then, differentiation of (10) in $t$ yields

$$
\dot{\mathbf{r}}_{1}+\dot{\Pi}_{1} \gamma_{1}+\Pi_{1} \gamma_{1}^{\prime} \frac{\mathrm{d} s}{\mathrm{~d} t}=\dot{\mathbf{r}}_{m}+\dot{\Pi}_{0} \gamma_{01}+\Pi_{0} \gamma_{01}^{\prime} \frac{\mathrm{d} s}{\mathrm{~d} t} .
$$

Since $\Pi_{1} \gamma_{1}^{\prime}=\overline{\mathbf{b}}_{1}$ and $\Pi_{0} \gamma_{01}^{\prime}=\overline{\mathbf{b}}_{01},(13)$ is reduced to, according to (12),

$$
\left(\dot{\mathbf{r}}_{1}-\dot{\mathbf{r}}_{m}\right)+\dot{\Pi}_{1} \gamma_{1}=\dot{\Pi}_{0} \gamma_{01},
$$

which from (9) can be written in the form

$$
\left(\dot{\mathbf{r}}_{1}-\dot{\mathbf{r}}_{m}\right)+\Pi_{1} \Omega_{1} \gamma_{1}-\Pi_{0} \Omega_{0} \gamma_{01}=0 .
$$

It should be remarked at this stage that the position constraint expressed by (10) is holonomic with two degreesof-freedom and the velocity constraint of (15) is Pfaffian with two DOFs, too. However, physical meanings of these 
two constraints are not directly connected to the constraint conditions based upon the assumptions of (1) and (2) mentioned in the previous section. In order to gain a physical insight into the rolling contact conditions, let us take the inner product of (15) and $\overline{\mathbf{b}}_{01}$ (or equivalently $\overline{\mathbf{b}}_{1}$ ), which results in

$$
R_{b 1}=\left(\dot{\mathbf{r}}_{1}-\dot{\mathbf{r}}_{m}\right)^{\mathrm{T}} \overline{\mathbf{b}}_{01}+\mathbf{b}_{1}^{\mathrm{T}} \Omega_{1} \gamma_{1}-\mathbf{b}_{01}^{\mathrm{T}} \Omega_{0} \gamma_{01}=0 .
$$

On account of the skew symmetry of $\Omega_{1}$ and $\Omega_{0}$ as shown in (9) and discussed below (9), (16) is reduced to

$$
R_{b 1}=\left(\dot{\mathbf{r}}_{1}-\dot{\mathbf{r}}_{m}\right)^{\mathrm{T}} \overline{\mathbf{b}}_{01}+\dot{p}_{1} \mathbf{n}_{1}^{\mathrm{T}} \gamma_{1}+\dot{\theta} \mathbf{n}_{01}^{\mathrm{T}} \gamma_{01}=0
$$

This shows the rolling contact constraint of a Pfaffian form as a condition of zero relative velocity of the contact point running on the fingertip relative to that of it running on the object. Another rolling contact constraint can be obtained by taking the inner product of (15) and $\overline{\mathbf{n}}_{01}$ in the following way:

$$
R_{n 1}=\left(\dot{\mathbf{r}}_{1}-\dot{\mathbf{r}}_{m}\right)^{\mathrm{T}} \overline{\mathbf{n}}_{01}-\mathbf{n}_{1}^{\mathrm{T}} \Omega_{1} \gamma_{1}-\mathbf{n}_{01}^{\mathrm{T}} \Omega_{0} \gamma_{01}=0,
$$

where $\Pi_{1} \mathbf{n}_{1}=-\Pi_{0} \mathbf{n}_{01}$ is taken into account. This is reduced to

$$
R_{n 1}=\left(\dot{\mathbf{r}}_{1}-\dot{\mathbf{r}}_{m}\right)^{\mathrm{T}} \overline{\mathbf{n}}_{01}+\dot{p}_{1} \mathbf{b}_{1}^{\mathrm{T}} \gamma_{1}-\dot{\theta} \mathbf{b}_{01}^{\mathrm{T}} \gamma_{01}=0
$$

Hence, if we define the column vectors

$$
\begin{gathered}
\mathfrak{R}_{b 1}=\left[\begin{array}{c}
-\overline{\mathbf{b}}_{01} \\
\left(\mathbf{n}_{01}^{\mathrm{T}} \gamma_{01}\right) \\
J_{1}^{\mathrm{T}}\left(q_{1}\right) \overline{\mathbf{b}}_{01}+\left(\mathbf{n}_{1}^{\mathrm{T}} \gamma_{1}\right) \mathbf{e}_{1}
\end{array}\right], \\
\mathfrak{R}_{n 1}=\left[\begin{array}{c}
-\overline{\mathbf{n}}_{01} \\
-\left(\mathbf{b}_{01}^{\mathrm{T}} \gamma_{01}\right) \\
J_{1}^{\mathrm{T}}\left(q_{1}\right) \overline{\mathbf{n}}_{01}+\left(\mathbf{b}_{1}^{\mathrm{T}} \gamma_{1}\right) \mathbf{e}_{1}
\end{array}\right],
\end{gathered}
$$

and $X_{1}=\left(\mathbf{x}^{\mathrm{T}}, \theta, q_{1}^{\mathrm{T}}\right)^{\mathrm{T}}, \mathbf{x}=(x, y)^{\mathrm{T}}$, and $J_{1}\left(q_{1}\right)=\partial \mathbf{r}_{1} / \partial q_{1}^{\mathrm{T}}$, then (17) and (19) can be rewritten into the forms

$$
R_{b 1}=\frac{\mathfrak{R}_{b 1}^{\mathrm{T}} \mathrm{d} X_{1}}{\mathrm{~d} t}=0, \quad R_{n 1}=\frac{\mathfrak{R}_{n 1}^{\mathrm{T}} \mathrm{d} X_{1}}{\mathrm{~d} t}=0 .
$$

A similar argument developed above can be applied to the characterization of the rolling contact constraint at the right-hand contact point $P_{2}$, at which the right-hand object contour and fingertip contour share the same tangent (see Figures 1 and 4). Therefore, it is possible to define smooth curves $\gamma_{2}\left(s_{2}\right)$ and $\gamma_{02}\left(s_{2}\right)$ expressed in their local coordinates $\mathrm{O}_{2}-\mathrm{X}_{2} Y_{2}$ and $\mathrm{O}_{m}-X Y$, respectively, with respect to the same arclength parameter $s_{2}$ as indicated in Figure 4. Note that in this paper the direction of increase of $s_{2}$ is taken to be counter to that of $s_{1}$. Hence, as shown in Figure 2 we see evidently that $\mathbf{b}_{2}=\mathrm{d} \gamma_{2} / \mathrm{d} s_{2}=\gamma_{2}^{\prime}$ and $\mathbf{b}_{02}=\mathrm{d} \gamma_{02} / \mathrm{d} s_{2}=\gamma_{02}^{\prime}$, and similarly to (10) and (12) that

$$
\begin{gathered}
\mathbf{r}_{2}+\Pi_{2} \gamma_{2}=\mathbf{r}_{m}+\Pi_{0} \gamma_{02}, \\
\overline{\mathbf{b}}_{2}=\Pi_{2} \mathbf{b}_{2}=\Pi_{0} \mathbf{b}_{02}=\overline{\mathbf{b}}_{02},
\end{gathered}
$$

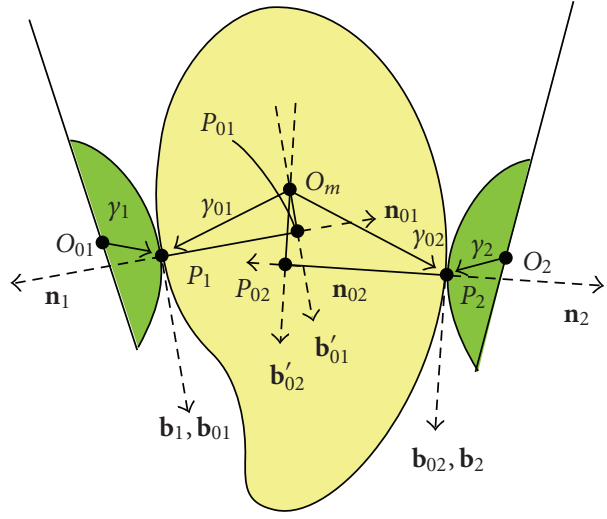

Figure 4: Definitions of tangent vectors $\mathbf{b}_{i}, \mathbf{b}_{0 i}$ and normals $\mathbf{n}_{i}$ and $\mathbf{n}_{0 i}$ at contact points $P_{i}$ for $i=1,2$.

where $\Pi_{2}=\left(\mathbf{r}_{X 2}, \mathbf{r}_{Y 2}\right), \dot{\Pi}_{2}=\Pi_{2} \Omega_{2}$, and

$$
\Omega_{2}=\dot{p}_{2}\left(\begin{array}{cc}
0 & 1 \\
-1 & 0
\end{array}\right)
$$

where $p_{2}=q_{21}+q_{22}$ according to the planar mechanism of the right-hand finger shown in Figure 1. Apparently from Figure 4 it follows that $\mathbf{b}_{2}^{\mathrm{T}} \Omega_{2}=-\dot{p}_{2} \mathbf{n}_{2}$ and $\mathbf{b}_{02}^{\mathrm{T}} \Omega_{0}=\dot{\theta} \mathbf{n}_{02}$. Thus, similarly to the derivation of (16) and (17), we have from differentiation of (22) in $t$ the following:

$$
\begin{aligned}
R_{b 2} & =\left(\dot{\mathbf{r}}_{2}-\dot{\mathbf{r}}_{m}\right)^{\mathrm{T}} \overline{\mathbf{b}}_{02}+\mathbf{b}_{2}^{\mathrm{T}} \Omega_{2} \gamma_{2}-\mathbf{b}_{02}^{\mathrm{T}} \Omega_{0} \gamma_{02}=0, \\
R_{b 2} & =\left(\dot{\mathbf{r}}_{2}-\dot{\mathbf{r}}_{m}\right)^{\mathrm{T}} \overline{\mathbf{b}}_{02}-\dot{p}_{2} \mathbf{n}_{2}^{\mathrm{T}} \gamma_{2}-\dot{\theta} \mathbf{n}_{02}^{\mathrm{T}} \gamma_{02}=0 .
\end{aligned}
$$

On account of the equality $\Pi_{2} \mathbf{n}_{2}=-\Pi_{0} \mathbf{n}_{02}$ as shown in Figures 1 and 4, we have, similarly to (18) and (19), the following:

$$
\begin{gathered}
R_{n 2}=\left(\dot{\mathbf{r}}_{2}-\dot{\mathbf{r}}_{m}\right)^{\mathrm{T}} \overline{\mathbf{n}}_{02}-\mathbf{n}_{2}^{\mathrm{T}} \Omega_{2} \gamma_{2}-\mathbf{n}_{02}^{\mathrm{T}} \Omega_{0} \gamma_{02}=0, \\
R_{n 2}=\left(\dot{\mathbf{r}}_{2}-\dot{\mathbf{r}}_{m}\right)^{\mathrm{T}} \overline{\mathbf{n}}_{02}-\dot{p}_{2} \mathbf{b}_{2}^{\mathrm{T}} \gamma_{2}+\dot{\theta} \mathbf{b}_{02}^{\mathrm{T}} \gamma_{02}=0 .
\end{gathered}
$$

Hence, if we denote $X=\left(\mathbf{x}^{\mathrm{T}}, \theta, q_{1}^{\mathrm{T}}, q_{2}^{\mathrm{T}}\right)^{\mathrm{T}}, J_{2}\left(q_{2}\right)=\partial \mathbf{r}_{2} / \partial q_{2}$, $\mathbf{e}_{2}=(1,1)^{\mathrm{T}}$, and

$$
\begin{gathered}
\mathfrak{R}_{b 2}=\left[\begin{array}{c}
-\overline{\mathbf{b}}_{02} \\
-\mathbf{n}_{02}^{\mathrm{T}} \gamma_{02} \\
0_{3} \\
J_{2}^{\mathrm{T}}\left(q_{2}\right) \overline{\mathbf{b}}_{02}-\left(\mathbf{n}_{2}^{\mathrm{T}} \gamma_{2}\right) \mathbf{e}_{2}
\end{array}\right], \\
\mathfrak{R}_{n 2}=\left[\begin{array}{c}
-\overline{\mathbf{n}}_{02} \\
\mathbf{b}_{02}^{\mathrm{T}} \gamma_{02} \\
0_{3} \\
J_{2}^{\mathrm{T}}\left(q_{2}\right) \overline{\mathbf{n}}_{02}-\left(\mathbf{b}_{2}^{\mathrm{T}} \gamma_{2}\right) \mathbf{e}_{2}
\end{array}\right],
\end{gathered}
$$


then, similarly to (21), (25) and (27) are expressed as follows:

$$
R_{b 2}=\frac{\mathfrak{R}_{b 2}^{\mathrm{T}} \mathrm{d} X}{\mathrm{~d} t}=0, \quad R_{n 2}=\frac{\mathfrak{R}_{n 2}^{\mathrm{T}} \mathrm{d} X}{\mathrm{~d} t}=0,
$$

where $0_{3}=(0,0,0)^{\mathrm{T}}$. For convenience, we append $\mathfrak{R}_{b 1}$ and $\mathfrak{R}_{n 1}$ with the two-dimensional zero vector in the following way:

$$
\overline{\mathfrak{R}}_{b 1}=\left[\begin{array}{c}
\mathfrak{R}_{b 1} \\
0_{2}
\end{array}\right], \quad \overline{\mathfrak{R}}_{n 1}=\left[\begin{array}{c}
\mathfrak{R}_{n 1} \\
0_{2}
\end{array}\right]
$$

and denote $\bar{\Re}_{b 1}$ and $\bar{\Re}_{n 1}$ by $\Re_{b 1}$ and $\Re_{n 1}$ renewedly for the sake of convenience. Then, (21) can be written in the form

$$
R_{b 1}=\frac{\Re_{b 1}^{\mathrm{T}} \mathrm{d} X}{\mathrm{~d} t}=0, \quad R_{n 1}=\frac{\mathfrak{R}_{n 1}^{\mathrm{T}} \mathrm{d} X}{\mathrm{~d} t}=0 .
$$

\section{Derivation of Euler-Lagrange Equation of Motion and Update Law of Length Parameters}

As discussed in the previous papers [11-15], a set of all possible postures of the fingers/object system depicted in Figure 1 can be regarded as a Riemannian manifold $M_{8}$ with eight degrees of freedom that can be represented by $M_{8}=$ $\left(\mathbf{E}^{2} \times S^{1}\right) \times T^{3} \times T^{2}$, where $(\mathbf{x}, \theta) \in \mathbf{E}^{2} \times S^{1}, q_{1} \in T^{3}$, $q_{2}=\left(q_{21}, q_{22}\right)^{\mathrm{T}} \in T^{2}, p_{2}=q_{2}^{\mathrm{T}} \mathbf{e}_{2}, \mathbf{e}_{2}=(1,1)^{\mathrm{T}}$, and the Riemannian metric $G=\left(g_{i j}\right)$ is defined as

$$
\begin{aligned}
K(X, \dot{X}) & =\frac{1}{2} \dot{X}^{\mathrm{T}} G(X) \dot{X} \\
& =\frac{M}{2}\left(\dot{x}^{2}+\dot{y}^{2}\right)+\frac{I}{2} \dot{\theta}^{2}+\sum_{i=1,2} \dot{q}_{i}^{\mathrm{T}} G_{i}\left(q_{i}\right) \dot{q}_{i}, \\
G & =\operatorname{diag}\left(M, M, I, G_{1}\left(q_{1}\right), G_{2}\left(q_{2}\right)\right),
\end{aligned}
$$

where $M$ and $I$ denote the mass of the object and its inertia moment around $O_{m}$, and $G_{i}\left(q_{i}\right)$ the inertia matrix of finger $i$ for $i=1,2$. Particularly, a set of all postures during rolling motions of the system keeping both rolling contacts can be regarded as a subset of $M_{8}$ that is subject to the constraints of (10) and (22). From the Riemannian geometric point of view, at any posture $X$ of the system with rolling contacts specified by some arclength parameters $s_{1}$ and $s_{2}$, the four equalities of (21) and (31) give rise to an assignment of the tangent vector $\dot{X} \in T_{\left(X ; s_{1}, s_{2}\right)} M_{8}$, where we denote by $T_{X ; s_{1}, s_{2}} M_{8}$ the tangent space of $M_{8}$ at the given posture $\left(X ; s_{1}, s_{2}\right)$ having rolling contacts at contact points $P_{1}\left(s_{1}\right)$ and $P_{2}\left(s_{2}\right)$. That is, the tangent vector should be orthogonal to all four vectors $\mathfrak{R}_{b i}$ and $\Re_{n i}$ for $i=1,2$. Hence, by introducing four Lagrange's multipliers $\lambda_{i}$ and $f_{i}$ corresponding to $\mathfrak{R}_{b i}$ and $\mathfrak{R}_{n i}$ for $i=$ 1,2 , it is possible to derive the Euler-Lagrange equation of motion in the following way:

$$
G(X) \ddot{X}+\left(\frac{1}{2} \dot{G}+S\right) \dot{X}+\sum_{i=1,2}\left(f_{i} \Re_{n i}+\lambda_{i} \Re_{b i}\right)=u .
$$

In detail, (34) can be written as follows:

$$
\begin{aligned}
& M \ddot{\mathbf{x}}-f_{1} \overline{\mathbf{n}}_{01}-f_{2} \overline{\mathbf{n}}_{02}-\lambda_{1} \overline{\mathbf{b}}_{01}-\lambda_{2} \overline{\mathbf{b}}_{02}=0, \\
& \quad I \ddot{\theta}-f_{1}\left(\mathbf{b}_{01}^{\mathrm{T}} \gamma_{01}\right)+f_{2}\left(\mathbf{b}_{02}^{\mathrm{T}} \gamma_{02}\right)+\lambda_{1}\left(\mathbf{n}_{01}^{\mathrm{T}} \gamma_{01}\right) \\
& \quad-\lambda_{2}\left(\mathbf{n}_{02}^{\mathrm{T}} \gamma_{02}\right)=0, \\
& G_{i}\left(q_{i}\right) \ddot{q}_{i}+\left\{\frac{1}{2} \dot{G}_{i}\left(q_{i}\right)+S_{i}\left(q_{i}, \dot{q}_{i}\right)\right\} \dot{q}_{i} \\
& \quad+f_{i}\left\{J_{i}^{\mathrm{T}}\left(q_{i}\right) \overline{\mathbf{n}}_{0 i}-(-1)^{i}\left(\mathbf{b}_{i}^{\mathrm{T}} \gamma_{i}\right) \mathbf{e}_{i}\right\} \\
& \quad+\lambda_{i}\left\{J_{i}^{\mathrm{T}}\left(q_{i}\right) \overline{\mathbf{b}}_{0 i}-(-1)^{i}\left(\mathbf{n}_{i}^{\mathrm{T}} \gamma_{i}\right) \mathbf{e}_{i}\right\}=u_{i}, \quad i=1,2,
\end{aligned}
$$

in which symbol $u_{i}$ in (37) denotes a control signal treated as an external torque that can be exerted through finger joints of finger $i$. It should be remarked that all of equations (35) to (37) are characterized by length parameters $s_{1}$ and $s_{2}$ but only quantities of the first fundamental form of contour curves are involved in (35) to (37). It should be also noted that the posture of the object is governed by the first-order differential equation of (9). At the same time, it is important to find the update law of length parameters $s_{1}$ and $s_{2}$ through rolling contact motion of the system. In fact, note that, from the condition (1) of rolling contact mentioned in Section 1, $\overline{\mathbf{b}}_{0 i}=\overline{\mathbf{b}}_{i}$ at contact point $P_{i}$ for $i=1,2$. This also implies

$$
\frac{\mathrm{d}}{\mathrm{d} t} \overline{\mathbf{b}}_{0 i}=\frac{\mathrm{d}}{\mathrm{d} t} \overline{\mathbf{b}}_{i}, \quad i=1,2,
$$

which reduces to

$$
\dot{\Pi}_{0} \mathbf{b}_{0 i}+\Pi_{0} \kappa_{0 i}\left(s_{i}\right) \mathbf{n}_{0 i} \frac{\mathrm{d} s_{i}}{\mathrm{~d} t}=\dot{\Pi}_{i} \mathbf{b}_{i}+\Pi_{i} \kappa_{i}\left(s_{i}\right) \mathbf{n}_{i} \frac{\mathrm{d} s_{i}}{\mathrm{~d} t}, \quad i=1,2,
$$

where $\kappa_{i}\left(s_{i}\right)$ denotes the curvature of the fingertip contour curve for finger $i$ for $i=1,2, \kappa_{01}\left(s_{1}\right)$ the curvature of the left-hand object contour, and $\kappa_{02}\left(s_{2}\right)$ that of the right-hand object contour. Since $\overline{\mathbf{n}}_{0 i}=-\overline{\mathbf{n}}_{i}$ for both $i=1,2$, (39) is again reduced to

$$
\left(\kappa_{0 i}+\kappa_{i}\right) \overline{\mathbf{n}}_{0 i} \frac{\mathrm{d} s_{i}}{\mathrm{~d} t}=\Pi_{i} \Omega_{i} \mathbf{b}_{i}-\Pi_{0} \Omega_{0} \mathbf{b}_{0 i}, \quad i=1,2,
$$

Thus, the inner product of $\overline{\mathbf{n}}_{0 i}$ and (40) yields

$$
\left(\kappa_{0 i}+\kappa_{i}\right) \frac{\mathrm{d} s_{i}}{\mathrm{~d} t}=(-1)^{i}\left(\dot{\theta}-\dot{p}_{i}\right), \quad i=1,2,
$$

where we referred to the relation (as discussed below (9)):

$$
\Omega_{i} \mathbf{b}_{i}=(-1)^{i} \dot{p}_{i} \mathbf{n}_{i}, \quad \Omega_{0} \mathbf{b}_{0 i}=-(-1)^{i} \dot{\theta} \mathbf{n}_{0 i}, \quad i=1,2 .
$$

The set of Euler-Lagrange equations (35)-(37) of motion of the system should be integrated simultaneously, accompanied with integration of the first order nonlinear differential equation of (41). 


\section{Integrability of Pfaffian Forms of Rolling Contact Constraints}

A rolling contact constraint between two rigid bodies is expressed traditionally by an equality of two contact-point velocities at the common contact point running on the one rigid body and on another body. In the case of rolling contact motion as shown in Figure 1, such zero relative-velocity relation is given in the Pfaffian forms of (29) and (31). We are now in a position to show that all these four Pfaffian forms are integrable in the sense of Frobenius $[16,17]$. In fact, it is possible to prove the following.

Proposition 4.1. (1) Under the contact conditions expressed by (10) and (22), the following four geometrical relations are valid (see Figure 4):

$$
\begin{aligned}
& Q_{b i}=\left(\mathbf{r}_{i}-\mathbf{r}_{m}\right)^{\mathrm{T}} \overline{\mathbf{b}}_{0 i}+\mathbf{b}_{i}^{\mathrm{T}} \gamma_{i}-\mathbf{b}_{0 i}^{\mathrm{T}} \gamma_{0 i}=0, \quad i=1,2, \\
& Q_{n i}=\left(\mathbf{r}_{i}-\mathbf{r}_{m}\right)^{\mathrm{T}} \overline{\mathbf{n}}_{0 i}-\mathbf{n}_{i}^{\mathrm{T}} \gamma_{i}-\mathbf{n}_{0 i}^{\mathrm{T}} \gamma_{0 i}=0, \quad i=1,2 .
\end{aligned}
$$

(2) Each of the Pfaffian forms $R_{b i}$ and $R_{n i}$ for $i=1,2$ defined by (17), (19), (25), and (27) is integrable in $t$ in the following forms:

$$
\frac{\mathrm{d}}{\mathrm{d} t} Q_{b i}=R_{b i}, \quad \frac{\mathrm{d}}{\mathrm{d} t} Q_{n i}=R_{n i}, \quad i=1,2 .
$$

In fact, the inner product of (10) and $\overline{\mathbf{b}}_{01}\left(=\overline{\mathbf{b}}_{1}\right)$ leads to (43) for $i=1$ and that of (22) and $\overline{\mathbf{b}}_{02}\left(=\overline{\mathbf{b}}_{2}\right)$ does to (43) for $i=2$. The inner product of (10) and $\overline{\mathbf{n}}_{01}\left(=-\overline{\mathbf{n}}_{1}\right)$ leads to (44) for $i=1$ and that of (22) and $\overline{\mathbf{n}}_{02}$ does to (44) for $i=2$. The first equation of (45) can be derived in the following manner:

$$
\begin{aligned}
\frac{\mathrm{d}}{\mathrm{d} t} Q_{b i}= & \left(\dot{\mathbf{r}}_{i}-\dot{\mathbf{r}}_{m}\right)^{\mathrm{T}} \overline{\mathbf{b}}_{0 i}+\left(\mathbf{r}_{i}-\mathbf{r}_{m}\right)^{\mathrm{T}} \frac{\mathrm{d}}{\mathrm{d} t} \overline{\mathbf{b}}_{0 i} \\
& +\left(\mathbf{b}_{i}^{\mathrm{T}} \gamma_{i}^{\prime}-\mathbf{b}_{0 i}^{\mathrm{T}} \gamma_{0 i}^{\prime}\right) \frac{\mathrm{d} s_{i}}{\mathrm{~d} t}+\left\{\left(\mathbf{b}_{i}^{\prime}\right)^{\mathrm{T}} \gamma_{i}-\left(\mathbf{b}_{0 i}^{\prime}\right)^{\mathrm{T}} \gamma_{0 i}\right\} \frac{\mathrm{d} s_{i}}{\mathrm{~d} t} \\
= & \left(\dot{\mathbf{r}}_{i}-\dot{\mathbf{r}}_{m}\right)^{\mathrm{T}} \overline{\mathbf{b}}_{0 i}+\left(\mathbf{r}_{i}-\mathbf{r}_{m}\right)^{\mathrm{T}}\left\{\dot{\Pi}_{0} \mathbf{b}_{0 i}+\Pi_{0} \mathbf{b}_{0 i}^{\prime} \frac{\mathrm{d} s_{i}}{\mathrm{~d} t}\right\} \\
& +(1-1) \frac{\mathrm{d} s_{i}}{\mathrm{~d} t}+\left(\kappa_{i} \mathbf{n}_{i}^{\mathrm{T}} \gamma_{i}-\kappa_{0 i} \mathbf{n}_{0 i}^{\mathrm{T}} \gamma_{0 i}\right) \frac{\mathrm{d} s_{i}}{\mathrm{~d} t} \\
= & \left(\dot{\mathbf{r}}_{i}-\dot{\mathbf{r}}_{m}\right)^{\mathrm{T}} \overline{\mathbf{b}}_{0 i}-(-1)^{i} \dot{\theta}\left(\mathbf{r}_{i}-\mathbf{r}_{m}\right)^{\mathrm{T}} \overline{\mathbf{n}}_{0 i} \\
& +\left(\mathbf{r}_{i}-\mathbf{r}_{m}\right)^{\mathrm{T}} \kappa_{0 i} \overline{\mathbf{n}}_{0 i} \frac{\mathrm{d} s_{i}}{\mathrm{~d} t}+\left(\kappa_{i} \mathbf{n}_{i}^{\mathrm{T}} \gamma_{i}-\kappa_{0 i} \mathbf{n}_{0 i}^{\mathrm{T}} \gamma_{0 i}\right) \frac{\mathrm{d} s_{i}}{\mathrm{~d} t},
\end{aligned}
$$

where, in the derivation of the last equality, (42) is referred to. Furthermore, substituting $Q_{n i}=0$ expressed by (44) into the right-hand side of (46) yields

$$
\begin{aligned}
\frac{\mathrm{d}}{\mathrm{d} t} Q_{b i}= & \left(\dot{\mathbf{r}}_{i}-\dot{\mathbf{r}}_{m}\right)^{\mathrm{T}} \overline{\mathbf{b}}_{0 i}+\left(\kappa_{i} \mathbf{n}_{i}^{\mathrm{T}} \gamma_{i}-\kappa_{0 i} \mathbf{n}_{0 i}^{\mathrm{T}} \gamma_{0 i}\right) \frac{\mathrm{d} s_{i}}{\mathrm{~d} t} \\
& +\left\{-(-1)^{i} \dot{\theta}+\kappa_{0 i} \frac{\mathrm{d} s_{i}}{\mathrm{~d} t}\right\}\left(\mathbf{n}_{i}^{\mathrm{T}} \gamma_{i}+\mathbf{n}_{0 i}^{\mathrm{T}} \gamma_{0 i}\right) \\
= & \left(\dot{\mathbf{r}}_{i}-\dot{\mathbf{r}}_{m}\right)^{\mathrm{T}} \overline{\mathbf{b}}_{0 i}+\left(\kappa_{i}+\kappa_{0 i}\right) \mathbf{n}_{i}^{\mathrm{T}} \gamma_{i} \frac{\mathrm{d} s_{i}}{\mathrm{~d} t} \\
& -(-1)^{i} \dot{\theta}\left(\mathbf{n}_{i}^{\mathrm{T}} \gamma_{i}+\mathbf{n}_{0 i}^{\mathrm{T}} \gamma_{0 i}\right), \quad i=1,2 .
\end{aligned}
$$

Thus, by applying (41) to this equation, we have

$$
\begin{aligned}
\frac{\mathrm{d}}{\mathrm{d} t} Q_{b i}= & \left(\dot{\mathbf{r}}_{i}-\dot{\mathbf{r}}_{m}\right)^{\mathrm{T}} \overline{\mathbf{b}}_{0 i}+(-1)^{i}\left(\dot{\theta}-\dot{p}_{i}\right) \mathbf{n}_{i}^{\mathrm{T}} \gamma_{i} \\
& -(-1)^{i} \dot{\theta}\left(\mathbf{n}_{i}^{\mathrm{T}} \gamma_{i}+\mathbf{n}_{0 i}^{\mathrm{T}} \gamma_{0 i}\right) \\
= & \left(\dot{\mathbf{r}}_{i}-\dot{\mathbf{r}}_{m}\right)^{\mathrm{T}} \overline{\mathbf{b}}_{0 i}-(-1)^{i}\left\{\dot{p}_{i} \mathbf{n}_{i}^{\mathrm{T}} \gamma_{i}+\dot{\theta} \mathbf{n}_{0 i}^{\mathrm{T}} \gamma_{0 i}\right\} \\
= & R_{b i}=0, \quad i=1,2 .
\end{aligned}
$$

The second equality of (45) can be verified in a similar way.

From this proposition, it follows that the Euler-Lagrange equation of motion of the system can be derived by applying the variational principle to the Lagrangian of the system

$$
L\left(X ; s_{1}, s_{2}\right)=K(X, \dot{X})-\sum_{i=1,2}\left(f_{i} Q_{n i}+\lambda_{i} Q_{b i}\right),
$$

where $K$ denotes the total kinetic energy of the system. Note that $K(X, \dot{X})$ is independent of the shape parameters $s_{1}$ and $s_{2}$ but $Q_{n i}$ and $Q_{b i}$ are dependent on $s_{i}$ for $i=1,2$, respectively. The variational principle is written in this case in the following form:

$$
\int_{t_{0}}^{t_{1}}\left\{\delta L+u_{1}^{\mathrm{T}} \delta q_{1}+u_{2}^{\mathrm{T}} \delta q_{2}\right\} \mathrm{d} t=0
$$

from which the set of equations (35) to (37) follow straightforwardly with control torques $u_{i}$ through finger joints as follows:

$$
G(X) \ddot{X}+\left(\frac{1}{2} \dot{G}(X)+S(X, \dot{X})\right) \dot{X}+\sum_{i=1,2}\left(f_{i} \Re_{n i}+\lambda_{i} \Re_{b i}\right)=u,
$$

where $u=\left(0,0,0, u_{1}^{\mathrm{T}}, u_{2}^{\mathrm{T}}\right)^{\mathrm{T}}$. It should be noted that

$$
\frac{\partial}{\partial X} Q_{n i}=\Re_{n i}, \quad \frac{\partial}{\partial X} Q_{b i}=\Re_{b i}, \quad i=1,2 .
$$

Thus, (51) can be spelled out in the general form

$$
\begin{aligned}
G(X) \ddot{X} & +\left(\frac{1}{2} \dot{G}(X)+S(X, \dot{X})\right) \dot{X} \\
& +\sum_{i=1,2}\left(f_{i} \frac{\partial}{\partial X} Q_{n i}+\lambda_{i} \frac{\partial}{\partial X} Q_{b i}\right)=B\left(\begin{array}{l}
u_{1} \\
u_{2}
\end{array}\right),
\end{aligned}
$$

where $B$ denotes the $8 \times 5$ constant matrix defined as $B^{\mathrm{T}}=$ $\left(0_{3 \times 5}, I_{5}\right), 0_{3 \times 5}$ signifies the $3 \times 5$ zero matrix, and $I_{5}$ the $5 \times 5$ identity matrix. In such a representation of the forcing term, $B$ is called the driving matrix in robotics. 


\section{Coordinate Control for Stable Grasping and Morse-Lyapunov Function}

In order to design a control signal for establishing stable grasp from the practical standpoint, a family of control signals has been introduced, based upon the fingers-thumb opposability that is one of the functional characteristics of human hands as discussed in [18]. The family of controls is described by the form

$$
u_{i}=-c_{i} \dot{q}_{i}+(-1)^{i} \beta J_{i}^{\mathrm{T}}\left(q_{i}\right)\left(\mathbf{r}_{1}-\mathbf{r}_{2}\right),
$$

where $\beta$ stands for a position feedback gain common for $i=1,2$ with physical unit $[\mathrm{N} / \mathrm{m}]$. It should be noted that the signal of the right-hand side can be constructed by the realtime measurement data on joint angles and angular velocities of both the robot fingers together with positions $\mathbf{r}_{1}$ and $\mathbf{r}_{2}$ of the centers of the fingertips. In other words, the control signal of (54) should be real time computed as a feedback signal from the measurement data of variables of the intrinsic finger world seen from the multifingered hand side. That is, any information of the geometry of the object and measurement data of location the object mass center should not be referred to in (54), because objects to be grasped, that are changeable in the situation, must be regarded as a substance in the extrinsic world.

Now, substituting $u_{i}$ for $i=1,2$ into (53) or directly into (37), we obtain

$$
\begin{aligned}
G_{i} \ddot{q}_{i} & +\left\{\frac{1}{2} \dot{G}_{i}+S_{i}\right\} \dot{q}_{i}+c_{i} \dot{q}_{i}-(-1)^{i} \beta J_{i}^{\mathrm{T}}\left(\mathbf{r}_{1}-\mathbf{r}_{2}\right) \\
& +f_{i}\left\{J_{i}^{\mathrm{T}} \overline{\mathbf{n}}_{0 i}-(-1)^{i}\left(\mathbf{b}_{i}^{\mathrm{T}} \gamma_{i}\right) \mathbf{e}_{i}\right\} \\
& +\lambda_{i}\left\{J_{i}^{\mathrm{T}} \overline{\mathbf{b}}_{0 i}-(-1)^{i}\left(\mathbf{n}_{i}^{\mathrm{T}} \gamma_{i}\right) \mathbf{e}_{i}\right\}=0, \quad i=1,2 .
\end{aligned}
$$

We call the set of equations (35), (36), and (55) the closedloop dynamics of the system. Clearly, the sum of inner products of $\dot{x}$ and (35), $\dot{\theta}$ and (36), and $\dot{q}_{i}$ and (55) for $i=1,2$ yields, due to (30) and (31),

$$
\frac{\mathrm{d}}{\mathrm{d} t}\left\{K(X, \dot{X})+\frac{\beta}{2}\left\|\mathbf{r}_{1}-\mathbf{r}_{2}\right\|^{2}\right\}=-\sum_{i=1,2} c_{i}\left\|\dot{q}_{i}\right\|^{2},
$$

where $K(X, \dot{X})$ denotes the total kinetic energy of the system defined in (32). It should be noted that the total energy defined by

$$
E(X, \dot{X})=K(X, \dot{X})+U(X)
$$

where

$$
U(X)=\frac{\beta}{2}\left\|\mathbf{r}_{1}-\mathbf{r}_{2}\right\|^{2},
$$

is nonnegative definite in $X$ and $\dot{X}$ and its time derivative $\mathrm{d} E / \mathrm{d} t$ is nonpositive definite as shown in (56). That is, if we regard the pair of position and velocity vectors $(X, \dot{X})$ as the state vector, then the quadratic function $E(X, \dot{X})$ looks like a Lyapunov function as seen from the energy relation (56).

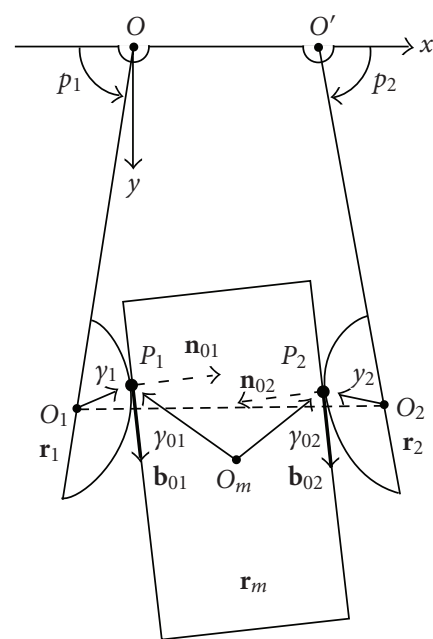

Figure 5: Precision prehension of a 2D object with parallel flat surfaces by a pair of single-DOF finger.

Nevertheless, in the case of the overall fingers/object system shown in Figure 1, $X=\left(\mathbf{x}^{\mathrm{T}}, \theta, q_{1}^{\mathrm{T}}, q_{2}^{\mathrm{T}}\right)^{\mathrm{T}}$ is of 8 dimentions and hence the state is of 16-dimension. Since there are four rolling contact constraints as discussed in Sections 3 and 4, the overall system must be of four degrees-of-freedom and therefore the state $(X, \dot{X})$ must be of 8 dimensions. However, the total energy function $E(X, \dot{X})$ is not positive definite with respect to $(X, \dot{X})$, because $U(X)$ is not positive definite in $X$ even if all of the four rolling contact constraints are taken into account. Thus, stabilization of grasping by means of a coordinate control of (54) cannot be treated by applying the conventional Lyapunov method to the relation of (56).

In order to tackle a control problem of stable grasping from the standpoint as a combination of Riemannian geometry and Lyapunov's direct method, we first consider a simpler case that the object has a pair of flat side surfaces that are parallel as shown in Figure 5 and both the robot fingers are of a single degree-of-freedom. In this case, $X=\left(\mathbf{x}^{\mathrm{T}}, \theta, q_{1}, q_{2}\right)^{\mathrm{T}}$ is of 5 dimensions and hence the overall fingers/object system is of single degrees-of-freedom. Therefore, the total energy $E(X, \dot{X})$ must be positive definite in $(X, \dot{X})$ under the four holonomic rolling contact constraints, because the scalar function $U\left(=(\beta / 2)\left\|\mathbf{r}_{1}-\mathbf{r}_{2}\right\|^{2}\right)$ must have a minimum at some posture $X=X^{*}$ under the constraints. That is,

$$
U_{m}=U\left(X^{*}\right)=\min U(X),
$$

where "min" is taken over all possible postures that are subject to rolling motion. For the sake of convenience, we will discuss the details of such a set of all possible postures reachable from a starting posture of the system by only movements of rolling motion. For the time being, we discuss what condition specifies the minimal posture that minimizes the artificial potential $U(X)$.

First, note that the difference vector $\mathbf{r}_{1}-\mathbf{r}_{2}$ can be expressed in terms of length parameters $s_{i}$ for $i=1,2$ as follows:

$$
\mathbf{r}_{1}-\mathbf{r}_{2}=-\Pi_{1} \gamma_{1}+\Pi_{2} \gamma_{2}+\Pi_{0}\left(\gamma_{01}-\gamma_{02}\right),
$$


which follows from subtraction of (22) from (10). When the object is rectangular as shown in Figure 5 , all $\mathbf{b}_{0 i}$ and $\mathbf{n}_{0 i}(i=$ $1,2)$ are invariant under the change of $s_{i}(i=1,2)$. As seen from Figure 5, if we denote the object width by $l_{w}$ then we have

$$
\begin{aligned}
\mathbf{r}_{1}-\mathbf{r}_{2}= & \left(s_{1}-s_{2}\right) \overline{\mathbf{b}}_{01}+\left(-\mathbf{b}_{1}^{\mathrm{T}} \gamma_{1}+\mathbf{b}_{2}^{\mathrm{T}} \gamma_{2}\right) \overline{\mathbf{b}}_{01} \\
& -l_{w} \overline{\mathbf{n}}_{01}+\left(\mathbf{n}_{1}^{\mathrm{T}} \gamma_{1}+\mathbf{n}_{2}^{\mathrm{T}} \gamma_{2}\right) \overline{\mathbf{n}}_{01} .
\end{aligned}
$$

Hence, by partially differentiating this equation in $s_{i}$, we obtain (the details will be given in the Appendix)

$$
\frac{\partial\left(\mathbf{r}_{1}-\mathbf{r}_{2}\right)}{\partial s_{i}}=(-1)^{i} \kappa_{i}\left(s_{i}\right)\left\{\left(\mathbf{n}_{i}^{\mathrm{T}} \gamma_{i}\right) \overline{\mathbf{b}}_{0 i}+\left(\mathbf{b}_{i}^{\mathrm{T}} \gamma_{i}\right) \overline{\mathbf{n}}_{0 i}\right\}, \quad i=1,2,
$$

where $\kappa_{i}\left(s_{i}\right)$ denotes the curvature of the fingertip contour for finger $i(i=1,2)$. Thus, by regarding $U(X)=U\left(s_{1}, s_{2}\right)$,

$$
\begin{array}{r}
\frac{\partial U\left(s_{1}, s_{2}\right)}{\partial s_{i}}=(-1)^{i} \beta \kappa_{i}\left(\mathbf{r}_{1}-\mathbf{r}_{2}\right)^{\mathrm{T}}\left\{\left(\mathbf{n}_{i}^{\mathrm{T}} \gamma_{i}\right) \overline{\mathbf{b}}_{0 i}+\left(\mathbf{b}_{i}^{\mathrm{T}} \gamma_{i}\right) \overline{\mathbf{n}}_{0 i}\right\} \\
i=1,2 .
\end{array}
$$

First, consider the simplest case when both the fingertips are spherical, which is called as the following:

Problem 1 (stability problem of ball-plate pinching). To find a necessary and sufficient condition under which minimization of the potential $U(X)$ in a set of all possible postures movable from a given starting posture by rolling motions is realized.

In this stabilization problem of ball-plate pinching, $O_{i}$ must be the center of the hemispheric fingertip of finger $i$ with radius $r_{i}=1 / \kappa_{i}\left(s_{i}\right)$, where $\kappa_{i}\left(s_{i}\right)$ is constant with respect to $s_{i}$ (see Figure 5 ). Hence, it follows that

$$
\mathbf{b}_{i}^{\mathrm{T}} \gamma_{i}=0, \quad \mathbf{n}_{i}^{\mathrm{T}} \gamma_{i}=r_{i}, \quad i=1,2 .
$$

Thus, in this case, (63) can be written in the form

$$
\frac{\partial U\left(s_{1}, s_{2}\right)}{\partial s_{i}}=(-1)^{i} \beta\left(\mathbf{r}_{1}-\mathbf{r}_{2}\right)^{\mathrm{T}} \overline{\mathbf{b}}_{0 i}, \quad i=1,2 .
$$

This means that $\partial U / \partial s_{i}=0$ arises if and only if $\left(\mathbf{r}_{1}-\mathbf{r}_{2}\right)$ is orthogonal to $\overline{\mathbf{b}}_{0 i}$ for $i=1,2$. In other words, $U\left(s_{1}, s_{2}\right)$ becomes extremal if and only if the four points $O_{1}, P_{1}, P_{2}$, and $\mathrm{O}_{2}$ are lying on the common straight line. At such a posture, it is easy to check that $s_{1}=s_{2}$ and the Hessian matrix $H=\left(\partial^{2} U / \partial s_{i} \partial s_{j}\right)$ becomes positive definite. Thus, we conclude that minimization of the shape function $U\left(s_{1}, s_{2}\right)$ is attained if and only if all $O_{1}, P_{1}, P_{2}$, and $O_{2}$ are lying on a straight line.

In the case that both the robot fingers are of a single degree-of-freedom mechanism as shown in Figure 5, the total degrees-of-freedom of the overall system becomes of one DOF, because originally the system has five DOFs but there are four holonomic constraints as discussed in
Sections 3 and 4. Hence, in this case, $U(X)$ becomes positive definite with respect to position variables under the four constraints. That is, $U(X)$ must be a Morse function introduced on a single-dimensional submanifold of the base Riemannian manifold $M=\left\{X, g_{i j}\right\}$ constrained by four holonomic constraints. Thus, the overall scalar function $E(X, \dot{X})$ defined by (57) becomes positive definite. This means that the equality relation of (56) shows that $E(X, \dot{X})$ can be regarded as a Lyapunov function for the closed-loop system (53) in which control inputs $u_{i}$ for $i=1,2$ defined by (54) are substituted. Therefore, the equilibrium point at which $\dot{X}=0$ and the four points $O_{1}, P_{1}, P_{2}$, and $O_{2}$ are on a common straight line becomes asymptotically stable in the sense of Lyapunov, even if the function $U(X)$ is defined on a Riemannian submanifold.

If each robot finger has multi-DOFs as shown in Figure 1, the overall fingers/object system becomes redundant in its degrees-of-freedom. For example, the left robot finger shown in Figure 1 has three joints and the right one has two joints. Then, the total degrees-of-freedom of the system becomes of four DOFs under the four holonomic constraints. Therefore, there arises an infinite number of possible postures realizing the minimum of potential $U(X)$; that is, a set of such possible postures themselves constitute a manifold. We call it an equilibrium manifold. In the case of a pair of robot fingers with two and three joints, respectively, pinching a rectangular object, the equilibrium manifold denoted by $E M^{3}$ becomes of three dimensions. In order to avoid a possible occurence of abundant motion (it is called a self-motion in robotics [19]) owing to the redundancy in the system's DOF, we have adopted the following form of control signals (see [5]):

$$
\begin{array}{r}
u_{i}=-c_{i} \dot{q}_{i}+(-1)^{i} \beta J_{i}^{\mathrm{T}}\left(q_{i}\right)\left(\mathbf{r}_{1}-\mathbf{r}_{2}\right)-\alpha_{i}\left\{p_{i}(t)-p_{i}(0)\right\} \mathbf{e}_{i}, \\
i=1,2,
\end{array}
$$

where $\alpha_{i}$ denotes a positive constant, and

$$
p_{i}(t)=q_{i}^{\mathrm{T}}(t) \mathbf{e}_{i}, \quad i=1,2,
$$

and $\mathbf{e}_{1}=(1,1,1)^{\mathrm{T}}$ and $\mathbf{e}_{2}=(1,1)^{\mathrm{T}}$ in the case of a pair of robot fingers shown in Figure 1. Substituting the control inputs of (66) into (53) yields

$$
\begin{aligned}
G_{i} \ddot{q}_{i} & +\left\{\frac{1}{2} \dot{G}_{i}+S_{i}\right\} \dot{q}_{i}+c_{i} \dot{q}_{i}-(-1)^{i} \beta J_{i}^{\mathrm{T}}\left(\mathbf{r}_{1}-\mathbf{r}_{2}\right) \\
& +\alpha_{i}\left\{p_{i}-p_{i}(0)\right\} \mathbf{e}_{i}+f_{i}\left\{J_{i}^{\mathrm{T}} \overline{\mathbf{n}}_{0 i}-(-1)^{i}\left(\mathbf{b}_{i}^{\mathrm{T}} \gamma_{i}\right) \mathbf{e}_{i}\right\} \\
& +\lambda_{i}\left\{J_{i}^{\mathrm{T}} \overline{\mathbf{b}}_{0 i}-(-1)^{i}\left(\mathbf{n}_{i}^{\mathrm{T}} \gamma_{i}\right) \mathbf{e}_{i}\right\}=0, \quad i=1,2 .
\end{aligned}
$$

Regarding the closed-loop system of (68), (35), and (36) with four constraints (43) and (44), we have the energy relation

$$
\begin{aligned}
& \frac{\mathrm{d}}{\mathrm{d} t}\left[K(X, \dot{X})+\frac{\beta}{2}\left\|\mathbf{r}_{1}-\mathbf{r}_{2}\right\|^{2}+\sum_{i=1,2} \frac{\alpha_{i}}{2}\left\{p_{i}-p_{i}(0)\right\}^{2}\right] \\
& =-\sum_{i=1,2} c_{i}\left\|\dot{q}_{i}\right\|^{2} .
\end{aligned}
$$




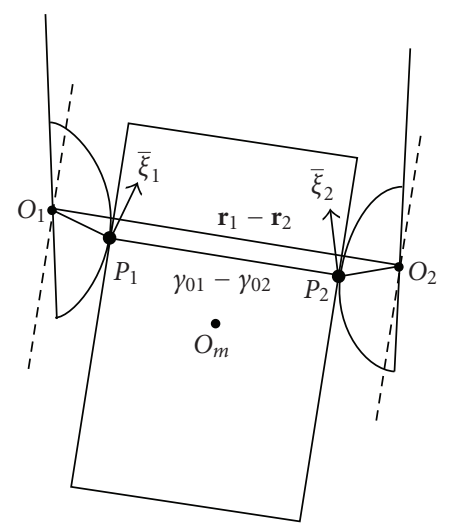

FIGURE 6: Minimization of the squared norm $\left\|\mathbf{r}_{1}-\mathbf{r}_{2}\right\|^{2}$ over rolling motions is attained when the straight line $\overline{P_{1} P_{2}}$ connecting the two contact points becomes parallel to the vector $\left(\mathbf{r}_{1}-\mathbf{r}_{2}\right)$; that is, $\overline{\mathrm{O}_{1} \mathrm{O}_{2}}$ becomes parallel to $\overline{P_{1} P_{2}}$.

The details of physical importance of the last term in the control signals of (66) will be disclosed in the next section when nonspherical fingertips are treated.

\section{Hessian Matrix of the Morse-Lyapunov Function}

Now we are in a position to discuss a stability problem of precision prehension (pinching) under the general fingertip geometry (nonspherical fingertip). However, we assume that the object is rectangular and both the curvatures $\kappa_{i}\left(s_{i}\right)$ for $i=1,2$ of the fingertips are continuously differentiable in $s_{i}$ and bounded between some $a(>0)$ and $b(>0)$ as shown in Figure 6 . The problem is posed as follows.

Problem 2 (stability problem of precision prehension). To find a necessary and sufficient conditon under which minimization of the potential $U(X)$ (or $\left.U\left(s_{1}, s_{2}\right)\right)$ is realized in a set of all movable postures starting from an initial posture by rolling contact motions. At the same time, to prove the asymptotic convergence of the orbit of motion to some possible equilibrium state.

First, as predicted reasonably from the analysis of Problem 1, we assume that, even in the general case of Problem 1 , all the three lines connecting $O_{1}$ and $P_{1}, P_{1}$ and $P_{2}$, and $\mathrm{P}_{2}$ and $\mathrm{O}_{2}$ are lying on the line $\left(\mathbf{r}_{1}-\mathbf{r}_{2}\right)$ for minimization of $\left\|\mathbf{r}_{1}-\mathbf{r}_{2}\right\|$. If it were true, then in the case of a pair of robot fingers with a single DOF (see Figure 5) the axes $\overline{\mathrm{OO}_{1}}$ and $\overline{\mathrm{O}^{\prime} \mathrm{O}_{2}}$ must be parallel and hence such a condition could not be satisfied in general, because the base points $O$ and $O^{\prime}$ are assumed to be fixed in the frame coordinates with some specified value of the length $\overline{O O^{\prime}}$.

Now, we treat a pair of robot fingers with multi DOFs as shown in Figure 1 and consider again a minimization problem of $U\left(s_{1}, s_{2}\right)$ together with a problem of finding a necessary condition for maintaining a steady state for the object (i.e., $\ddot{\mathbf{x}}=0$ in (35) and $\ddot{\theta}=0$ in (36)). First, to attain a steady state of the object, we need the conditions

$$
f_{1}=f_{2}=f_{d}(>0), \quad \lambda_{1}=-\lambda_{2}=\lambda_{d}
$$

to satisfy $\ddot{\mathbf{x}}=0$ in (35) since all $\overline{\mathbf{n}}_{0 i}$ and $\overline{\mathbf{b}}_{0 i}(i=1,2)$ are invariant in this case. Further, to satisfy $\ddot{\theta}=0$ in (36), we shall examine the conditions

$$
s_{1}=s_{2}, \quad \mathbf{b}_{1}^{\mathrm{T}} \gamma_{1}=\mathbf{b}_{2}^{\mathrm{T}} \gamma_{2} .
$$

Then, substituting these two equalities into (61) yields

$$
\begin{aligned}
\mathbf{r}_{1}-\mathbf{r}_{2} & =-l_{w} \overline{\mathbf{n}}_{01}+\left(\mathbf{n}_{1}^{\mathrm{T}} \gamma_{1}+\mathbf{n}_{2}^{\mathrm{T}} \gamma_{2}\right) \overline{\mathbf{n}}_{01} \\
& =-\left(l_{w}-\mathbf{n}_{1}^{\mathrm{T}} \gamma_{1}-\mathbf{n}_{2}^{\mathrm{T}} \gamma_{2}\right) \overline{\mathbf{n}}_{01},
\end{aligned}
$$

which shows that $\left(\mathbf{r}_{1}-\mathbf{r}_{2}\right)$ must be perpendicular to $\overline{\mathbf{b}}_{01}$ (= $\overline{\mathbf{b}}_{02}$ ). It is also reasonable to show that, under the equality conditions of (71), $\ddot{\mathbf{x}}=0$ and $\ddot{\theta}=0$ can be expressed as

$$
\begin{gathered}
-f_{1} \overline{\mathbf{n}}_{01}-f_{2} \overline{\mathbf{n}}_{02}-\lambda_{1} \overline{\mathbf{b}}_{01}-\lambda_{2} \overline{\mathbf{b}}_{02}=0, \\
-f_{1}\left(\mathbf{b}_{01}^{\mathrm{T}} \gamma_{01}\right)+f_{2}\left(\mathbf{b}_{02}^{\mathrm{T}} \gamma_{02}\right)+\lambda_{1}\left(\mathbf{n}_{01}^{\mathrm{T}} \gamma_{01}\right)-\lambda_{2}\left(\mathbf{n}_{02}^{\mathrm{T}} \gamma_{02}\right)=0 .
\end{gathered}
$$

In fact, the first equality of (73) follows from (71) and $\overline{\mathbf{n}}_{01}=$ $-\overline{\mathbf{n}}_{02}$ and $\overline{\mathbf{b}}_{01}=\overline{\mathbf{b}}_{02}$ and the second equality follows in such a way that

$$
\begin{gathered}
-f_{d}\left\{\mathbf{b}_{01}^{\mathrm{T}}\left(\gamma_{01}-\gamma_{02}\right)\right\}-\lambda_{d}\left\{\mathbf{n}_{01}^{\mathrm{T}}\left(\gamma_{01}-\gamma_{02}\right)\right\} \\
=-\lambda_{d} \mathbf{n}_{01}^{\mathrm{T}}\left(\gamma_{01}-\gamma_{02}\right) .
\end{gathered}
$$

Hence, if $\lambda_{d}=0$, then the second equality of (73) becomes valid. Furthermore, in the case of Problem 2, $\mathbf{b}_{i}^{\mathrm{T}} \gamma_{i} \neq 0$ in general and hence (63) does not imply $\partial U / \partial s_{i}=0(i=1,2)$ though $\left(\mathbf{r}_{1}-\mathbf{r}_{2}\right)$ must be perpendicular to $\overline{\mathbf{b}}_{0 i}$. In this case, we must bear in mind that length parameters $s_{1}$ and $s_{2}$ are not independent to each other.

In order to derive the Hessian of the shape function $U\left(s_{1}, s_{2}\right)$ plus the extra term (the third term in the bracket [ ] of (69)) with respcet to $q_{1}, q_{2}$, and $\theta$, we put

$$
P(X)=\frac{\beta}{2}\left\|\mathbf{r}_{1}-\mathbf{r}_{2}\right\|^{2}+\sum_{i=1,2} \frac{\alpha_{i}}{2} \hat{N}_{i}^{2},
$$

where we define

$$
\widehat{N}_{i}=p_{i}-p_{i}(0), \quad i=1,2 .
$$

From (63), we see that the shape function $U\left(s_{1}, s_{2}\right)$ that expresses the first term of the right-hand side of (75) reduces to

$$
\begin{aligned}
U(X) & =\frac{\beta}{2}\left\|\mathbf{r}_{1}-\mathbf{r}_{2}\right\|^{2}=\frac{\beta}{2}\left\{d^{2}\left(s_{1}, s_{2}\right)+l^{2}\left(s_{1}, s_{2}\right)\right\} \\
& =U\left(s_{1}, s_{2}\right),
\end{aligned}
$$


where, by denoting the object width by $l_{w}$,

$$
\begin{gathered}
d\left(s_{1}, s_{2}\right)=s_{1}-s_{2}-\mathbf{b}_{1}^{\mathrm{T}} \gamma_{1}+\mathbf{b}_{2}^{\mathrm{T}} \gamma_{2}, \\
l\left(s_{1}, s_{2}\right)=-l_{w}+\left(\mathbf{n}_{1}^{\mathrm{T}} \gamma_{1}+\mathbf{n}_{2}^{\mathrm{T}} \gamma_{2}\right)
\end{gathered}
$$

(see Figures 4 and 6). First we show that

$$
d P=S_{N} d \theta+\sum_{i=1,2} \Delta N_{i} \mathbf{e}_{i}^{\mathrm{T}} d q_{i}
$$

where

$$
\begin{gathered}
S_{N}=\beta\left\{\left(s_{1}-s_{2}\right) l+l_{w} d\right\}, \\
\Delta N_{i}=\beta N_{i}+\alpha_{i} \hat{N}_{i}, \quad i=1,2, \\
N_{i}=(-1)^{i}\left(\mathbf{b}_{i}^{\mathrm{T}} \gamma_{i}\right) l-\left(\mathbf{n}_{i}^{\mathrm{T}} \gamma_{i}\right) d, \quad i=1,2,
\end{gathered}
$$

in which we omit writing $s_{1}$ and $s_{2}$ in $d\left(s_{1}, s_{2}\right)$ and $l\left(s_{1}, s_{2}\right)$ for simplicity. In fact, it follows from (63), (61), and (41) that

$$
\begin{aligned}
\frac{\mathrm{d}}{\mathrm{d} t} P= & \frac{\mathrm{d}}{\mathrm{d} t} U\left(s_{1}, s_{2}\right)+\sum_{i=1,2} \alpha_{i} \hat{N}_{i} \frac{\mathrm{d} \hat{N}_{i}}{\mathrm{~d} t} \\
= & \sum_{i=1,2}\left[(-1)^{i} \beta\left\{\left(\mathbf{n}_{i}^{\mathrm{T}} \gamma_{i}\right) d-(-1)^{i}\left(\mathbf{b}_{i}^{\mathrm{T}} \gamma_{i}\right) l\right\} \kappa_{i} \frac{\mathrm{d} p_{i}}{\mathrm{~d} t}\right. \\
& \left.\quad+\alpha_{i} N \frac{\mathrm{d} p_{i}}{\mathrm{~d} t}\right] \\
= & \sum_{i=1,2}\left[\beta\left\{\left(\mathbf{n}_{i}^{\mathrm{T}} \gamma_{i}\right) d-(-1)^{i}\left(\mathbf{b}_{i}^{\mathrm{T}} \gamma_{i}\right) l\right\}\left(\dot{\theta}-\dot{p}_{i}\right)+\alpha_{i} \hat{N}_{i} \dot{p}_{i}\right] \\
= & \beta\left\{\left(\mathbf{n}_{1}^{\mathrm{T}} \gamma_{1}+\mathbf{n}_{2}^{\mathrm{T}} \gamma_{2}\right) d+\left(\mathbf{b}_{1}^{\mathrm{T}} \gamma_{1}-\mathbf{b}_{2}^{\mathrm{T}} \gamma_{2}\right) l\right\} \dot{\theta} \\
& +\sum_{i=1,2}\left(\beta N_{i}+\alpha_{i} \hat{N}_{i}\right) \dot{p}_{i} \\
= & \beta\left\{\left(l+l_{w}\right) d+\left(s_{1}-s_{2}-d\right) l\right\} \dot{\theta}+\sum_{i=1,2} \Delta N_{i} \dot{p}_{i}
\end{aligned}
$$

where, in the last equality, (81) is used. By using (80), (83) reduces to (79). Equation (79) means that a local minimum of $P$ as a function $P\left(X, s_{1}, s_{2}\right)$ of $X$ and $s_{1}$ and $s_{2}$ is attained when $S_{N}=0$ and $\Delta N_{i}=0(i=1,2)$. This condition is satisfied when $\overline{\mathrm{O}_{1} \mathrm{O}_{2}}$ is parallel to $\overline{P_{1} P_{2}}$ and $\Delta N_{i}=0$. More explicitly, if we define

$$
\begin{gathered}
\Delta f_{i}=f_{i}+\beta l\left(s_{1}, s_{2}\right), \\
\Delta \lambda_{i}=\lambda_{i}-(-1)^{i} \beta d\left(s_{1}, s_{2}\right), \quad i=1,2,
\end{gathered}
$$

then the closed-loop dynamics of (35), (36), and (68) can be rewritten into

$$
\begin{aligned}
& M \ddot{\mathbf{x}}-\sum_{i=1,2}\left(\Delta f_{i} \overline{\mathbf{n}}_{0 i}+\Delta \lambda_{i} \overline{\mathbf{b}}_{0 i}\right)=0, \\
& I \ddot{\theta}+\sum_{i=1,2}(-1)^{i}\left\{\Delta f_{i}\left(\mathbf{b}_{0 i}^{\mathrm{T}} \gamma_{0 i}\right)-\Delta \lambda_{i}\left(\mathbf{n}_{0 i}^{\mathrm{T}} \gamma_{0 i}\right)\right\}+S_{N}=0,
\end{aligned}
$$

$$
\begin{aligned}
G \ddot{q}_{i}+ & \left\{\frac{1}{2} \dot{G}_{i}+S_{i}\right\} \dot{q}_{i}+c_{i} \dot{q}_{i}+\Delta N_{i} \mathbf{e}_{i} \\
& +\Delta f_{i}\left\{J_{i}^{\mathrm{T}} \overline{\mathbf{n}}_{0 i}-(-1)^{i}\left(\mathbf{b}_{i}^{\mathrm{T}} \gamma_{i}\right) \mathbf{e}_{i}\right\} \\
& +\Delta \lambda_{i}\left\{J_{i}^{\mathrm{T}} \overline{\mathbf{b}}_{0 i}-(-1)^{i}\left(\mathbf{n}_{i}^{\mathrm{T}} \gamma_{i}\right) \mathbf{e}_{i}\right\}=0, \quad i=1,2 .
\end{aligned}
$$

Apparently, steady states are attained when $S_{N}=0, \Delta N_{i}=0$, $\Delta f_{i}=0, \Delta \lambda_{i}=0$ for $i=1,2$.

Now, let us derive the Hessian matrix of $P\left(X, s_{1}, s_{2}\right)$ by calculating $\mathrm{d} S_{N} / \mathrm{d} t$ and $\mathrm{d} \Delta N_{i} / \mathrm{d} t$ for $i=1,2$. In a similar way to the derivation of (83), we obtain

$$
\begin{aligned}
& \frac{\mathrm{d}}{\mathrm{d} t} S_{N}=\left(v_{1}+v_{2}\right) \frac{\mathrm{d} \theta}{\mathrm{d} t}-v_{1} \frac{\mathrm{d} p_{1}}{\mathrm{~d} t}-v_{2} \frac{\mathrm{d} p_{2}}{\mathrm{~d} t}, \\
& \frac{\mathrm{d} \Delta N_{i}}{\mathrm{~d} t}=-v_{i} \frac{\mathrm{d} \theta}{\mathrm{d} t}+\left(\alpha_{i}+v_{i i}\right) \frac{\mathrm{d} p_{i}}{\mathrm{~d} t}-v_{i j} \frac{\mathrm{d} p_{i}}{\mathrm{~d} t},
\end{aligned}
$$

for $i \neq j$, where

$$
\begin{gathered}
v_{i}=-\beta l\left\{\frac{1}{\kappa_{i}}-\frac{l_{w}}{l} \mathbf{n}_{i}^{\mathrm{T}} \gamma_{i}\right\}, \\
v_{i i}=-\beta l\left\{\frac{1}{\kappa_{i}}+\mathbf{n}_{i}^{\mathrm{T}} \gamma_{i}-\frac{\left(\mathbf{n}_{i}^{\mathrm{T}} \gamma_{i}\right)^{2}+\left(\mathbf{b}_{i}^{\mathrm{T}} \gamma_{i}\right)^{2}}{l}\right\}, \\
v_{i j}=-\beta\left\{\left(\mathbf{n}_{1}^{\mathrm{T}} \gamma_{1}\right)\left(\mathbf{n}_{2}^{\mathrm{T}} \gamma_{2}\right)-\left(\mathbf{b}_{1}^{\mathrm{T}} \gamma_{1}\right)\left(\mathbf{b}_{2}^{\mathrm{T}} \gamma_{2}\right)\right\},
\end{gathered}
$$

for $i=1,2$ and $j \neq i$. Note that $l<0$ by definition, and all $v_{1}$, $v_{2}, v_{11}$, and $v_{22}$ are positive provided that

$$
\frac{1}{\kappa_{i}}>-\mathbf{n}_{i}^{\mathrm{T}} \gamma_{i}, \quad i=1,2 .
$$

Thus, if we denote $\mathbf{z}=\left(\theta, p_{1}, p_{2}\right)^{\mathrm{T}}$, then we obtain

$$
\begin{gathered}
\frac{\partial P}{\partial \mathbf{z}}=\left(\frac{\partial P}{\partial \theta}, \frac{\partial P}{\partial p_{1}}, \frac{\partial P}{\partial p_{2}}\right)^{\mathrm{T}}=\left(S_{N}, \Delta N_{1}, \Delta N_{2}\right)^{\mathrm{T}} \\
\frac{\partial^{2} P}{\partial \mathbf{z} \partial \mathbf{z}^{\mathrm{T}}}=\left[\begin{array}{ccc}
\frac{\partial^{2} P}{\partial \theta^{2}} & \frac{\partial^{2} P}{\partial \theta \partial p_{1}} & \frac{\partial^{2} P}{\partial p_{1} \partial p_{2}} \\
\frac{\partial^{2} P}{\partial p_{1} \partial \theta} & \frac{\partial^{2} P}{\partial p_{1}^{2}} & \frac{\partial^{2} P}{\partial p_{1} \partial p_{2}} \\
\frac{\partial^{2} P}{\partial p_{2} \partial \theta} & \frac{\partial^{2} P}{\partial p_{2} \partial p_{1}} & \frac{\partial^{2} P}{\partial p_{2}^{2}}
\end{array}\right] \\
= \\
\left.\begin{array}{ccc}
v_{1}+v_{2} & -v_{1} & -v_{2} \\
-v_{1} & \alpha_{1}+v_{11} & -v_{12} \\
-v_{2} & -v_{21} & \alpha_{2}+v_{22}
\end{array}\right) .
\end{gathered}
$$


It is possible to verify that the Hessian of $P$ with respect to $\mathbf{z}$ in a neighborhood of the posture satisfying $\partial P / \partial \mathbf{z}=0$ is positive definite by choosing $\alpha_{i}>$ appropriately so as to satisfy $\alpha_{i}>-\beta l / \kappa_{i}$ for $i=1,2$ (note that $\left.l<0\right)$. Finally, the Hessian of $P$ with respect to the position state vector $X$ is given by

$$
\begin{aligned}
H_{X} & =\frac{\partial^{2} P}{\partial X \partial X^{\mathrm{T}}}=\left(\frac{\partial \mathbf{z}^{\mathrm{T}}}{\partial X}\right) \frac{\partial^{2} P}{\partial \mathbf{z} \partial \mathbf{z}^{\mathrm{T}}}\left(\frac{\partial \mathbf{z}}{\partial X^{\mathrm{T}}}\right) \\
& =D^{\mathrm{T}}\left(\frac{\partial^{2} P}{\partial \mathbf{z} \partial \mathbf{z}^{\mathrm{T}}}\right) D=D^{\mathrm{T}} H_{\mathbf{z}} D,
\end{aligned}
$$

where $D$ is a constant $3 \times 8$-matrix of the form

$$
D=\left(\begin{array}{llllllll}
0 & 0 & 1 & 0 & 0 & 0 & 0 & 0 \\
0 & 0 & 0 & 1 & 1 & 1 & 0 & 0 \\
0 & 0 & 0 & 0 & 0 & 0 & 1 & 1
\end{array}\right),
$$

because $\mathbf{z}$ can be expressed by $\mathbf{z}=D X$. Thus, the Hessian matrix $H_{X}$ is degenerate, but it is possible to see that the function $P(X)$ can be regarded as a Morse-Bott function (see [20]).

\section{A Proof of Dirichlet-Lagrange Stability for Precision Prehension}

We define the equilibrium manifold by the set of all postures that have a form depicted in Figure 6 and maintain the contacts at some points $P_{1}$ and $P_{2}$. More rigorously, this equilibrium manifold denoted by $E M^{3}$ can be regarded as a set of all of $X$ that satisfies $S_{N}=0, \Delta N_{1}=0$, and $\Delta N_{2}=0$ under the situation that $p_{i}(0)$ for $i=1,2$ are given. The submanifold $E M^{3}$ is of 3 dimensions, because it satisfies $\partial P / \partial \mathbf{z}=0$ and maintains both contacts (i.e., the 2-dimensional contact constraints (12) and (22)) that determine automatically the position of the object mass center. In other words, $E M^{3}$ corresponds to a set of motions of the three excess degrees-of-freedom of the robot fingers.

Suppose one posture $X^{*}$ of the system belonging to the submanifold $E M^{3}$ and a Riemannian ball $B\left(X^{*}, r_{0}\right)$ that is defined by

$$
B\left(X^{*}, r_{0}\right)=\left\{X X \in\left\{M, \bar{g}_{i j}\right\}, d\left(X^{*}, X\right)<r_{0}\right\},
$$

where $d\left(X^{*}, X\right)$ signifies the Riemannian distance defined by

$$
d\left(X^{*}, X\right)=\inf _{X(\tau)} \int_{0}^{t} \sqrt{\sum \bar{g}_{i j} \dot{X}_{i}(\tau) \dot{X}_{j}(\tau)} \mathrm{d} \tau,
$$

where the infimum is taken over all of the orbits in $\left\{M, \bar{g}_{i j}\right\}$ connecting $X^{*}$ and $X$ from $\tau=0$ to $\tau=t$. We denote the $i j$-entry of $G(X)$ introduced in (32) and (33) by $\bar{g}_{i j}$. We will show that, for any $\varepsilon>0$, there exists $\delta(\varepsilon)>0$ such that any solution trajectory $X(t)$ as a solution to the closedloop dynamics of (85) to (87) starting from any posture $X(0)$ with rest state inside $B\left(X^{*}, \delta(\varepsilon)\right)$ remains in $B\left(X^{*}, \varepsilon\right)$ and converges asymptotically to some posture on $E M^{3}$ as $t \rightarrow \infty$. Note that numerical values of $r_{0}, \varepsilon$, and $\delta(\varepsilon)$ are given on the basis of physical dimension $[\sqrt{\mathrm{kg}} \mathrm{m}]$, that is, the dimension of the Riemannian distance defined in (96). We split the tangent space $T_{X} M$ into the horizontal space spanned from the constraints of (28) and (30) and the kernel space as the orthogonal complement to the horizontal space. This is carried out by defining

$$
\begin{gathered}
\Phi=\left(Q_{n 1}, Q_{b 1}, Q_{n 2}, Q_{b 2}\right)^{\mathrm{T}}, \\
\Phi_{X}^{\mathrm{T}}=\frac{\partial \Phi^{\mathrm{T}}}{\partial X}=\left(\Re_{n 1}, \Re_{b 1}, \Re_{n 2}, \Re_{b 2}\right), \\
\Delta \Lambda=\left(\Delta f_{1}, \Delta \lambda_{1}, \Delta f_{2}, \Delta \lambda_{2}\right)^{\mathrm{T}}
\end{gathered}
$$

and rewriting (85) to (87) into the following general form similarly to (53):

$$
G(X) \ddot{X}+\left(\frac{1}{2} \dot{G}(X)+S(X, \dot{X})\right) \dot{X}+\Phi_{X}^{\mathrm{T}} \Delta \Lambda+C \dot{X}+\frac{\partial}{\partial X} P=0,
$$

where $C=\operatorname{diag}\left(0,0,0, c_{1} I_{3}, c_{2} I_{2}\right)$. We bear in mind that $\partial P / \partial X=D^{\mathrm{T}}(\partial P / \partial \mathbf{z})$ according to $(91)$ and $(94)$. Then, let us introduce the transformation

$$
\begin{gathered}
\dot{X}=\left(\Phi_{X}^{\mathrm{T}}\left(\Phi_{X} \Phi_{X}^{\mathrm{T}}\right)^{-1 / 2}, \Psi\right)\left(\begin{array}{c}
\dot{\Phi} \\
\dot{\boldsymbol{\eta}}
\end{array}\right), \\
W=\left(\Phi_{X}^{\mathrm{T}}\left(\Phi_{X} \Phi\right)^{-1 / 2}, \Psi\right),
\end{gathered}
$$

where $\Psi$ is an $8 \times 4$-matrix whose column vectors have a unit norm, and orthogonal to each other and to every column vector of $\Phi_{X}^{\mathrm{T}}$. That is, $W$ is an orthogonal matrix and hence

$$
W^{\mathrm{T}}=\left(\begin{array}{c}
\left(\Phi_{X} \Phi_{X}^{\mathrm{T}}\right)^{-1 / 2} \Phi_{X} \\
\Psi^{\mathrm{T}}
\end{array}\right)=W^{-1} .
$$

Since $\dot{\Phi}$ must be the null vector, substituting (99) into (98) yields

$$
\bar{G} \ddot{\boldsymbol{\eta}}+\left(\frac{1}{2} \dot{\bar{G}}+\bar{S}\right) \dot{\boldsymbol{\eta}}+\left(\Psi^{\mathrm{T}} C \Psi\right) \dot{\boldsymbol{\eta}}+\Psi^{\mathrm{T}} D^{\mathrm{T}} \frac{\partial P}{\partial z}=0,
$$

where $\bar{G}=\Psi^{\mathrm{T}} G \Psi$, and

$$
\bar{S}=\Psi^{\mathrm{T}} S \Psi-\frac{1}{2} \dot{\Psi}^{\mathrm{T}} G \Psi+\frac{1}{2} \Psi^{\mathrm{T}} G \dot{\Psi} .
$$

It should be noted that $\bar{S}$ is skew symmetric, too. It is also important to note that the dynamics of (98) appear to be not fully dissipated according to the definition $C$ mentioned below (98). Nevertheless, from (17) and (25) it follows that

$$
\left(\dot{\mathbf{r}}_{1}-\dot{\mathbf{r}}_{2}\right) \overline{\mathbf{n}}_{01}+\dot{p}_{1} \mathbf{n}_{1}^{\mathrm{T}} \gamma_{1}+\dot{p}_{2} \mathbf{n}_{2}^{\mathrm{T}} \gamma_{2}+\dot{\theta}\left(\mathbf{n}_{01}^{\mathrm{T}} \gamma_{01}+\mathbf{n}_{02}^{\mathrm{T}} \gamma_{02}\right)=0 .
$$

Since the coefficient of $\dot{\theta}$ is $-l_{w}$, (103) reduces to

$$
l_{w}^{2} \dot{\theta}^{2} \leq c_{\theta 1}\left\|\dot{q}_{1}\right\|^{2}+c_{\theta 2}\left\|\dot{q}_{2}\right\|^{2}
$$


with positive constants $c_{\theta 1}$ and $c_{\theta 2}$. Similarly, we see from (19) and (27) that

$$
\begin{gathered}
\left(\dot{\mathbf{r}}_{1}+\dot{\mathbf{r}}_{2}\right)^{\mathrm{T}} \overline{\mathbf{n}}_{01}-2 \dot{\mathbf{r}}_{m}^{\mathrm{T}} \overline{\mathbf{n}}_{01}+\dot{p}_{1} \mathbf{b}_{1}^{\mathrm{T}} \gamma_{1}+\dot{p}_{2} \mathbf{b}_{2}^{\mathrm{T}} \gamma_{2} \\
-\dot{\theta}\left(\mathbf{b}_{01}^{\mathrm{T}} \gamma_{01}+\mathbf{b}_{02}^{\mathrm{T}} \gamma_{02}\right)=0 .
\end{gathered}
$$

From (25) and (17) we also have

$$
\begin{gathered}
\left(\dot{\mathbf{r}}_{1}+\dot{\mathbf{r}}_{2}\right)^{\mathrm{T}} \overline{\mathbf{n}}_{01}-2 \dot{r}_{m}^{\mathrm{T}} \bar{b}_{01}+\dot{p}_{1} \mathbf{n}_{1}^{\mathrm{T}} \gamma_{1}-\dot{p}_{2}^{\mathrm{T}} \mathbf{n}_{2}^{\mathrm{T}} \gamma_{2} \\
-\dot{\theta}\left(\mathbf{n}_{01}^{\mathrm{T}} \gamma_{01}+\mathbf{n}_{02}^{\mathrm{T}} \gamma_{02}\right)=0 .
\end{gathered}
$$

Since $\dot{\mathbf{r}}_{m}=(\dot{x}, \dot{y})$ and $\left(\overline{\mathbf{n}}_{01}, \overline{\mathbf{b}}_{01}\right) \in S O(2)$, it follows from (104) and (105) that

$$
\|\dot{\mathbf{x}}\|^{2} \leq c_{01}\left\|\dot{q}_{1}\right\|^{2}+c_{02}\left\|\dot{q}_{2}\right\|^{2}+l_{w}^{2} \dot{\theta}^{2}
$$

with some constants $\mathcal{c}_{01}>0$ and $c_{02}>0$. Inequalities (104) and (107) imply that the damping coefficient matrix $\bar{C}=$ $\Psi^{\mathrm{T}} C \Psi$ in (101) is positive definite, and hence the dynamics of (101) are fully dissipated. On the other hand, the inner product of (101) and $\dot{\boldsymbol{\eta}}$ yields

$$
\frac{\mathrm{d}}{\mathrm{d} t}(K(X, \dot{\boldsymbol{\eta}})+P)=-\dot{\boldsymbol{\eta}}^{\mathrm{T}} \bar{C} \dot{\boldsymbol{\eta}}
$$

where

$$
K(X, \dot{\boldsymbol{\eta}})=\frac{1}{2} \dot{\boldsymbol{\eta}}^{\mathrm{T}} \bar{G} \dot{\boldsymbol{\eta}}=\frac{1}{2} \dot{X}^{\mathrm{T}} G(X) \dot{X}=K(X, \dot{X}),
$$

where $K(X, \dot{X})$ is defined by (32). It is also important to note that the coefficient matrix $J^{\mathrm{T}}=\Psi^{\mathrm{T}} D^{\mathrm{T}}$ of $\partial P / \partial \mathbf{z}$ is nondegenerate (in any regular position of grasping like Figure 1) and hence it is possible to construct a scalar quantity

$$
V=\left(\Delta \theta, \Delta p_{1}, \Delta p_{2}\right)^{\mathrm{T}} J^{+} \bar{G} \dot{\boldsymbol{\eta}}
$$

where $J^{+}=J^{\mathrm{T}}\left(J J^{\mathrm{T}}\right)^{-1}$. Then, in a similar way to the proof of stability of PD feedback for redundant systems discussed previously in [8] and [13], we find a constant $\alpha>0$ and another constant $\sigma>0$ by choosing $\beta>0, \alpha_{i}>0, c_{i}>0$ (i= 1,2 ) adequately such that

$$
\begin{aligned}
\frac{1-\alpha}{1+\alpha} E(X, \dot{\boldsymbol{\eta}}) & \leq E(X, \dot{\boldsymbol{\eta}})+\alpha V \\
& \leq \frac{1+\alpha}{1-\alpha} E(X, \dot{\boldsymbol{\eta}}), \\
\frac{\mathrm{d}}{\mathrm{d} t} W(X, \dot{\boldsymbol{\eta}}, \alpha) & \leq-\sigma W(X, \dot{\boldsymbol{\eta}}, \alpha),
\end{aligned}
$$

where $W(X, \dot{\boldsymbol{\eta}}, \alpha)=E(X, \dot{\boldsymbol{\eta}})+\alpha V$. The speed of convergence depends on $\sigma$ depending on $\alpha$, but eventually it can be regulated by the choice of control gains $\beta, \alpha_{i}, c_{i}(i=1,2)$. Thus, the asymptotic stability of some $X^{*}$ on the equilibrium manifold $E M^{3}$ is ensured in the sense of Riemannian distance. However, the design problem of such control gains according to the physical scale of a multifingered hand must be important from the robotics research viewpoint but has not yet been fully investigated.

\section{Conclusions}

A mathematical modeling of 2-dimensional grasping of an object by a pair of robot fingers under rolling contact constraints is presented under the situation that the geometric shape of both the fingertips and 2D object is arbitrary. In a special class of rigid objects having a pair of parallel planar surfaces, a general class of control signals is proposed, which can be constructed only from the robot finger kinematics without sensing the object. An extension of the Dirichlet-Lagrange stability theorem to the case of precision prehension under the existence of rolling contact constraints and redundancy in system's degrees-of-freedom is discussed. A preliminary result on computer simulation is presented in [21]. Finally, it should be remarked that any extension of stabilization control for precision prehension to the case of arbitrary shape objects has not yet been tackled.

\section{Appendix}

In (61), note that $\overline{\mathbf{b}}_{01}\left(=\overline{\mathbf{b}}_{02}\right)$ and $\overline{\mathbf{n}}_{01}\left(=\overline{\mathbf{n}}_{02}\right)$ are invariant under the change of $s_{i}(i=1,2)$. Hence, by partially differentiating the right-hand side of (61) in $s_{1}$, we obtain

$$
\begin{aligned}
\frac{\partial\left(\mathbf{r}_{1}-\mathbf{r}_{2}\right)}{\partial s_{1}}= & \overline{\mathbf{b}}_{01}+\left(-\kappa_{1} \mathbf{n}_{1}^{\mathrm{T}} \gamma_{1}-\mathbf{b}_{1}^{\mathrm{T}} \gamma_{1}^{\prime}\right) \overline{\mathbf{b}}_{01} \\
& +\left(-\kappa_{1} \mathbf{b}_{1}^{\mathrm{T}} \gamma_{1}+\mathbf{n}_{1}^{\mathrm{T}} \gamma_{1}^{\prime}\right) \overline{\mathbf{n}}_{01} \\
= & -\kappa_{1}\left\{\left(\mathbf{n}_{1}^{\mathrm{T}} \gamma_{1}\right) \overline{\mathbf{b}}_{01}+\left(\mathbf{b}_{1}^{\mathrm{T}} \gamma_{1}\right) \overline{\mathbf{n}}_{01}\right\},
\end{aligned}
$$

since $\gamma_{1}^{\prime}=\partial \gamma_{1} / \partial s_{1}=\mathbf{b}_{1}$. It is important to remark that the content of \{\} in (A.1) dentoed by $\bar{\xi}_{1}$ as in (78) can be depicted as a vector $\bar{\xi}_{1}$ shown in Figure 6, which is the vector $\overrightarrow{O_{1} P_{1}}$ itself $\left(=\Pi_{1} \gamma_{1}\right)$ rotated by angle $\pi / 2$ in the frame coordinates. Similarly, we have

$$
\frac{\partial\left(\mathbf{r}_{1}-\mathbf{r}_{2}\right)}{\partial s_{2}}=\kappa_{2}\left\{\left(\mathbf{n}_{2}^{\mathrm{T}} \gamma_{2}\right) \overline{\mathbf{b}}_{02}+\left(\mathbf{b}_{2}^{\mathrm{T}} \gamma_{2}\right) \overline{\mathbf{n}}_{02}\right\} .
$$

\section{References}

[1] D. J. Montanna, "The kinematics of contact and grasps," International Journal of Robotics Research, vol. 7, no. 3, pp. 1732, 1988.

[2] D. J. Montanna, "Contact stability for two-fingered grasps," IEEE Transactions on Robotics and Automation, vol. 8, no. 4, pp. 421-430, 1992.

[3] A. B. A. Cole, J. E. Hauser, and S. S. Sastry, "Kinematics and control of multifingered hands with rolling contact," IEEE Transactions on Automatic Control, vol. 34, no. 4, pp. 398-404, 1989.

[4] N. Sarkar, X. Yun, and V. Kumar, "Dynamic control of 3-D rolling contacts in two-arm manipulation," IEEE Transactions on Robotics and Automation, vol. 13, no. 3, pp. 364-376, 1997.

[5] S. Arimoto, "Intelligent control of multi-fingered hands," Annual Reviews in Control, vol. 28, no. 1, pp. 75-85, 2004.

[6] S. Arimoto, "A differential-geometric approach for 2D and 3D object grasping and manipulation," Annual Reviews in Control, vol. 31, no. 2, pp. 189-209, 2007. 
[7] R. M. Murray, Z. Li, and S. S. Sastry, A Mathematical Introduction to Robotic Manipulation, CRC Press, Boca Raton, Fla, USA, 1994.

[8] S. Arimoto, M. Yoshida, M. Sekimoto, and K. Tahara, "A Riemannian-geometry approach for dynamics and control of object manipulation under constraints," SICE Journal of Control, Measurement, and System Integration, vol. 2, no. 2, pp. 107-116, 2009.

[9] K. Nomizu, "Kinematics and differential geometry of submanifolds_rolling a ball with a prescribed locus of contact," Tohoku Mathematical Journal, vol. 30, pp. 623-637, 1978.

[10] A. Gray, E. Abbena, and S. Salamon, Modern Differential Geometry of Curves and Surfaces with Mathematica, Chapman \& Hall/CRC, Boca Raton, Fla, USA, 2006.

[11] S. Arimoto, M. Sekimoto, M. Yoshida, and K. Tahara, "Modeling and control of 2-D grasping of an object with arbitrary shape under rolling contact," SICE Journal of Control, Measurement, and System Integration, vol. 2, no. 6, pp. 379386, 2009.

[12] S. Arimoto, M. Yoshida, M. Sekimoto, K. Tahara, and J.-H. Bae, "Modeling and control for 2-D grasping of an object with arbitrary shape under rolling contact," in Proceedings of the 9th International IFAC Symposium on Robot Control (SYROCO '09), pp. 517-522, Gifu, Japan, September 2009.

[13] S. Arimoto, M. Yoshida, M. Sekimoto, and K. Tahara, "A Riemannian-geometry approach for dynamics and control of object manipulation under constraints," in Proceedings of the IEEE International Conference on Robotics and Automation, pp. 1683-1690, Kobe, Japan, May 2009.

[14] S. Arimoto, M. Yoshida, M. Sekimoto, and K. Tahara, "A Riemannian-geometry approach for modeling and control of dynamics of object manipulation under constraints," International Journal of Robotics Research, vol. 2009, Article ID 892801, 16 pages, 2009.

[15] S. Arimoto, "Modeling and control of multi-body mechanical systems: part I A Riemannian geometry approach," International Journal of Factory Automation, Robotics and Soft Computing, no. 2, pp. 108-122, 2009.

[16] W. M. Oliva, Geometric Mechanics, vol. 1798 of Lecture Notes in Mathemaics, Springer, Berlin, Germany, 2002.

[17] F. Bullo and A. D. Lewis, Geometric Control of Mechanical Systems, Springer, New York, NY, USA, 2005.

[18] J. Napier, Hands, Princeton University Press, Princeton, NJ, USA, 1993.

[19] H. Seraji, "Configuration control of redundant manipulators: theory and implimentation," IEEE Transactions on Robotics and Automation, vol. 5, no. 4, pp. 403-420, 1987.

[20] R. Bott, "The stable homotopy of the classical groups," Annals of Mathematics, vol. 70, no. 2, pp. 313-337, 1959.

[21] M. Yoshida, S. Arimoto, and K. Tahara, "Manipulation of 2D object with arbitrary shape by two robot finger under rolling constraint," in Proceedings of the ICROS-SICE International Joint Conference 2009, pp. 695-699, Fukuoka, Japan, August 2009. 

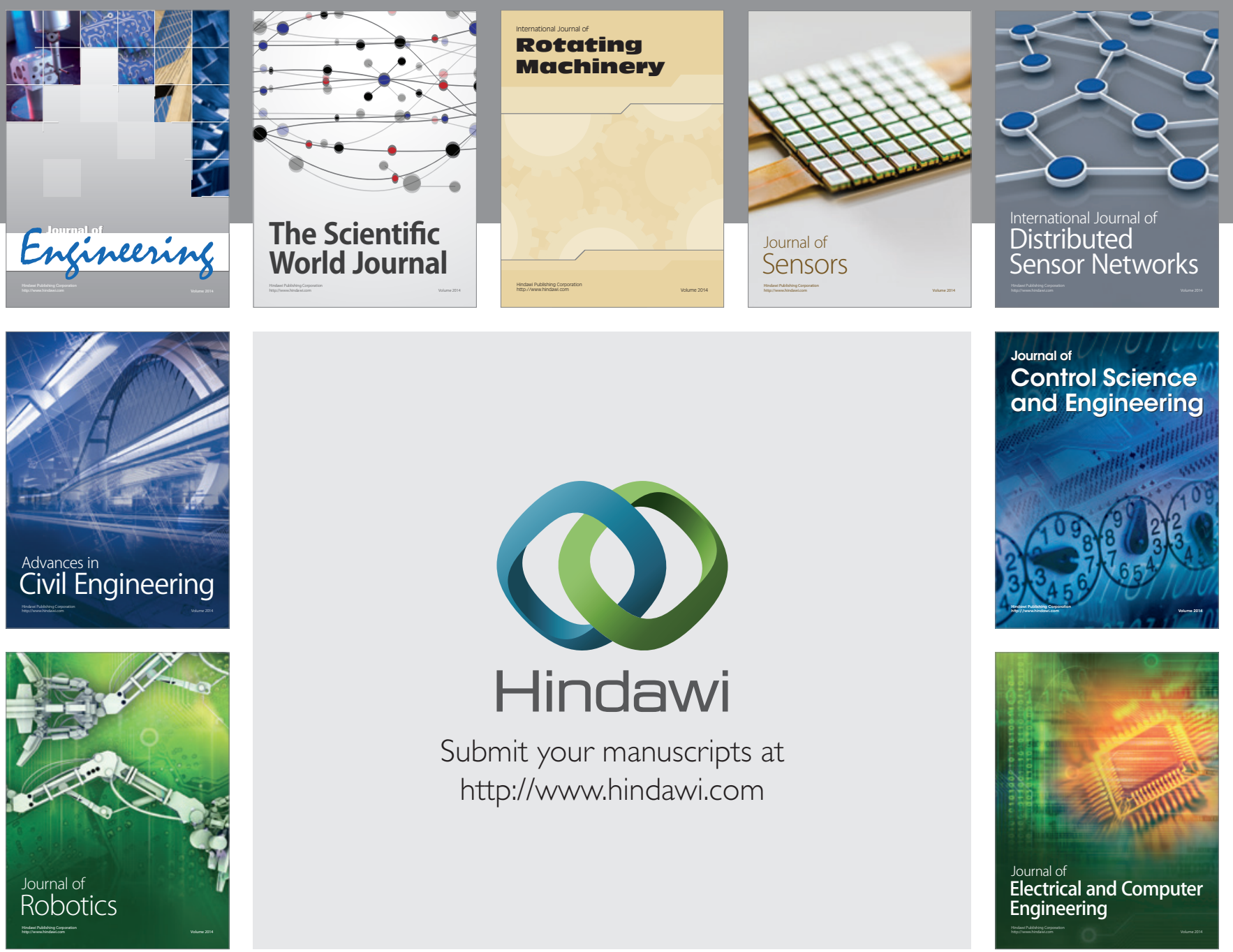

Submit your manuscripts at

http://www.hindawi.com
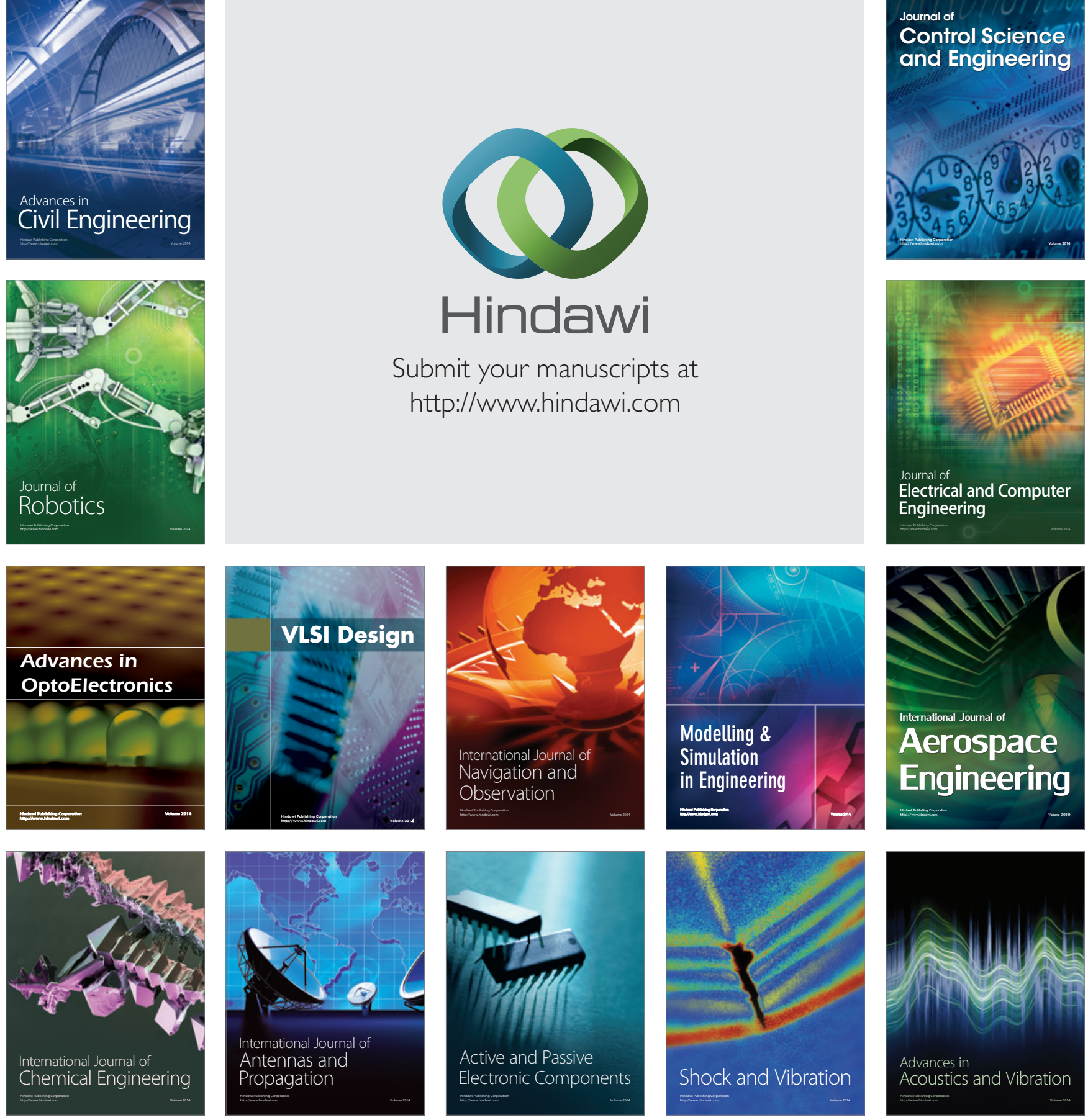